Florida International University FIU Digital Commons

\title{
General Elementary Music Teacher Burn-out in Miami-Dade County Public Schools
}

Luciano Alberto

Florida International University, Lucianoz28@hotmail.com

DOI: $10.25148 /$ etd.FI1 1042101

Follow this and additional works at: https://digitalcommons.fiu.edu/etd

\section{Recommended Citation}

Alberto, Luciano, "General Elementary Music Teacher Burn-out in Miami-Dade County Public Schools" (2011). FIU Electronic Theses and Dissertations. 351.

https://digitalcommons.fiu.edu/etd/351

This work is brought to you for free and open access by the University Graduate School at FIU Digital Commons. It has been accepted for inclusion in FIU Electronic Theses and Dissertations by an authorized administrator of FIU Digital Commons. For more information, please contact dcc@fiu.edu. 


\title{
FLORIDA INTERNATIONAL UNIVERSITY
}

Miami, Florida

\section{GENERAL ELEMENTARY MUSIC TEACHER BURN-OUT IN MIAMI-DADE COUNTY PUBLIC SCHOOLS}

\author{
A thesis submitted in partial fulfillment of the \\ requirements for the degree of \\ MASTER OF SCIENCE \\ in \\ MUSIC EDUCATION \\ by \\ Luciano Alberto
}

2011 


\section{To: Dean Brian Schriner}

College of Architecture and the Arts

This thesis, written by Luciano Alberto and entitled General Elementary Music Teacher Burn-out in Miami-Dade County Public Schools, having been approved in respect to style and intellectual content, is referred to you for your judgment.

We have read this thesis and recommend that it be approved.

Catherine Rand

Erskine Dottin

Janet Duguay Kirsten, Major Professor

Date of Defense: March 21, 2011

The thesis of Luciano Alberto is approved.

Dean Brian Schriner

College of Architecture and the Arts

Interim Dean Kevin O’Shea

University Graduate School

Florida International University, 2011 


\title{
ABSTRACT OF THE THESIS \\ GENERAL ELEMENTARY MUSIC TEACHER BURN-OUT IN MIAMI-DADE COUNTY PUBLIC SCHOOLS
}

\author{
by \\ Luciano Alberto \\ Florida International University, 2011 \\ Miami, Florida \\ Professor Janet Duguay Kirsten, Major Professor
}

This study sought to determine whether there is a significant difference in the level of burn-out between general elementary music educators employed by Miami-Dade County Public Schools and general elementary music educators from the rest of Florida. Burnout can be defined as a state where a professional feels completely fatigued, dehumanized, reduced in regard to achievement, chronic despair, and withdrawn (Dworkin, 1987). Previous studies of this syndrome have used a Maslach Burn-out Inventory (M.B.I) to assess burn-out indicators. This study examined morale in the current educational atmosphere, using a modified M.B.I. to identify differences between two sample sets: one from Miami-Dade County Public Schools and another from other Florida counties. Applying the Mann-Whitney U test to the results revealed significant differences between the two populations on four items. In conclusion, areas of high stress for teachers were related to the FCAT, administrative support, and job security. 


\section{TABLE OF CONTENTS}

CHAPTER

PAGE

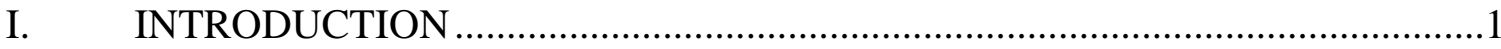

Research Purpose and Significance of the Study..............................................

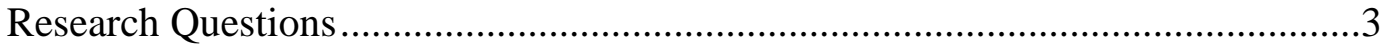

Delimitations Regarding M-DCPS Elementary Music Educators and Attrition ....3

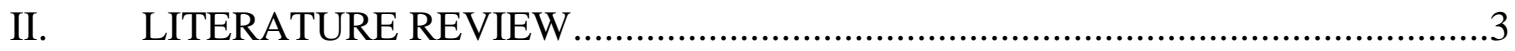

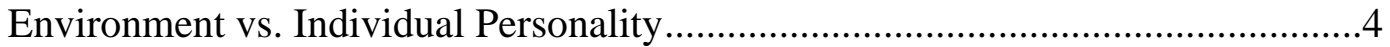

Music Teachers and Burn-Out ............................................................................6

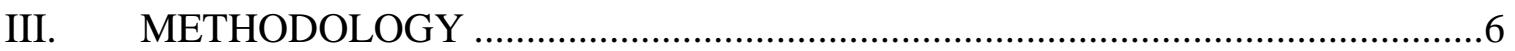

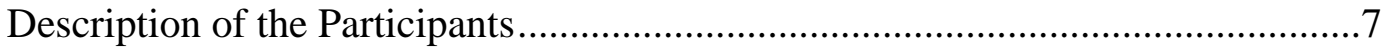

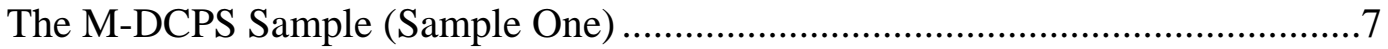

Other Florida Counties Sample (Sample Two).................................................8

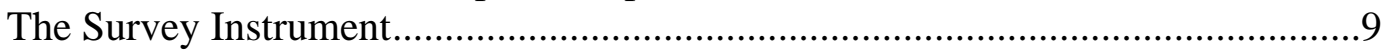

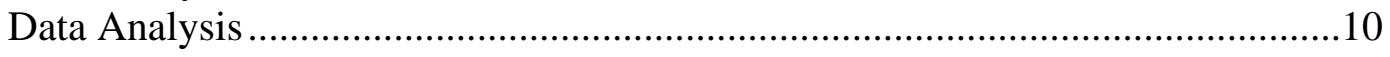

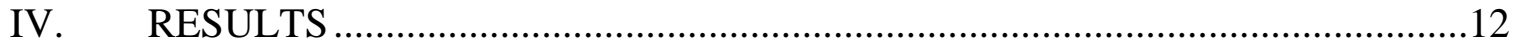

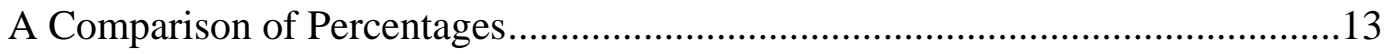

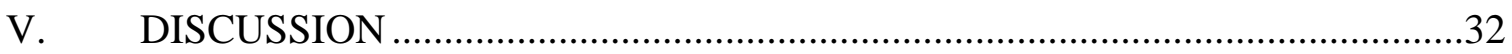

Qualitative Self-Reported Perceptions ...............................................................33

Reducing the Effects of Stress and Burn-out....................................................33

Approaches to Lifestyle Changes ...............................................................33

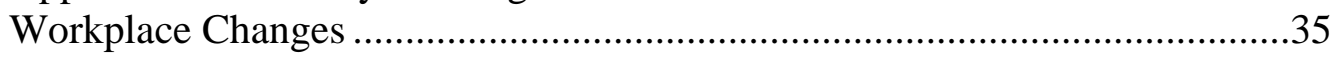

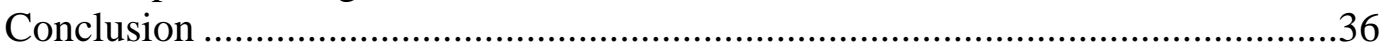

LIST OF REFERENCES......................................................

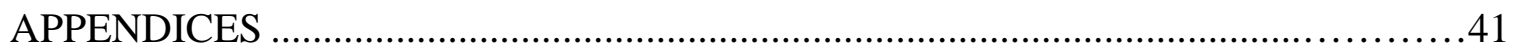




\section{$/, 67$ F TABLES}

TABLE

PAGE

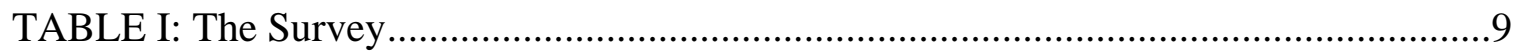

TABLE II: Test Statistics (Mann-Whitney U/Wilcoxon W) ........................................11

TABLE III: Comparative Frequencies for Samples One and Two.................................13

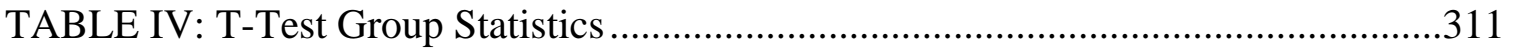

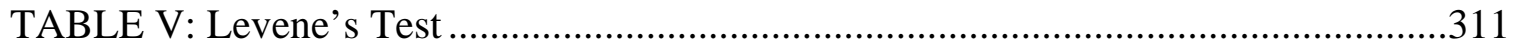




\section{LIST OF FIGURES}

FIGURE

PAGE

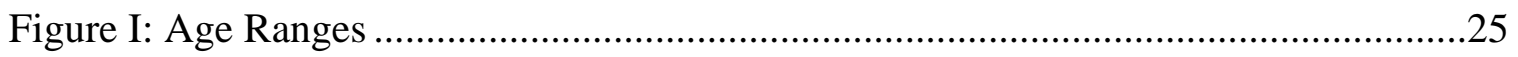

Figure II: School Aged Children.....................................................................................25

Figure III: Years of Experience ………………………….....................................26

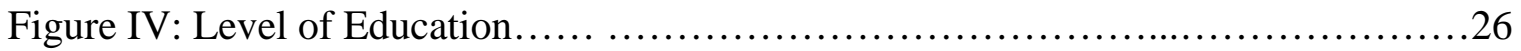

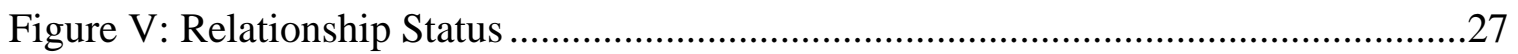

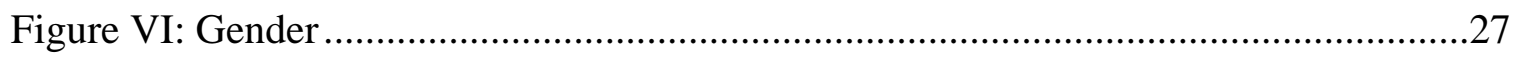

Figure VII: Overall Burn-Out Scores for Both Samples ....................................................28

Figure VIII: Q-Q Plots for Both Samples .....................................................................29

Figure IX: Total Burn-Out Score by Sample Box Plot.......................................................30 


\section{CHAPTER I \\ INTRODUCTION}

The purpose of this study was to identify unique conditions currently affecting elementary music educators in Miami-Dade County Public Schools (M-DCPS) as compared to elementary music educators from the rest of Florida. It is important to identify major factors of workplace stress (stressors) that ultimately lead to burn-out among Florida teachers. This study will also identify remedies to improve the retention of music teachers in M-DCPS.

Research Purpose and Significance of the Study

The intent of this study is to examine conditions affecting elementary music educators in M-DCPS and their levels of burn-out, as compared to the rest of Florida. One representative sample was taken from attendees of the Florida Music Educators National conference (FMENC) in Tampa, Florida in January 2011. The second representative sample consisted of randomly selected M-DCPS general music educators. Results of this study identified levels of teacher burn-out and remedies to improve retention of Florida music educators.

Teacher burn-out is creating a void that will eventually cause a shortage of experienced music educators in M-DCPS. This shortage could be exacerbated by the current economic crisis and the imminent wave of baby-boomer retirements. M-DCPS has recently offered "four plan options" to many older teachers as incentives for early retirement for the purpose of budgetary reductions (OPPAGA, 2007). According to the Office of Program Policy Analysis and Governance Accountability (OPPAGA), 10\% of Miami-Dade teachers left the field in 2007 (OPPAGA, 2007). OPPAGA identified 
several circumstances that contribute to a trend towards increased teacher attrition, including dissatisfaction (30\%) and retirement (35\%). Additionally, another 23\% of teachers cited personal reasons for leaving, which could mask symptoms of burn-out, as professionals are maybe reluctant to admit to feelings of inadequacy (OPPAGA, 2007).

The term "revolving door" is commonly used to define the trend that as more experienced teachers leave the field, less experienced teachers, ill-prepared to cope with the myriad challenges the profession poses, fill their positions (Dworkin, 1987). Professionally speaking, teaching loads have recently increased for music educators. As budget problems have forced M-DCPS to set aside funds to continue normal operations, the district has reallocated educators. In many instances, these educators now service two school sites instead of one. During the 2008-2009 school year, funds ran so low that MDCPS requested employees to work two days without immediate compensation (McGrory \& Samuels, 2009). Music education professionals currently face an uncertainty in relation to job security. This situation is potentially comparable to that of the last major economic depression in the United States, when a survey of 700 schools, taken by the Office of Education, found that 35 cities had eliminated music programs altogether (Efland, 1983). There is a fear that without change, M-DCPS music programs could be deemed unnecessary and taken completely out of the curriculum.

Teacher burn-out is a multifaceted problem in M-DCPS due to geographical location, economic conditions (South Florida has been affected especially hard because educational funding has heavily depended on the housing sector), and the size of the county (M-DCPS is the fourth largest county in the nation). Music educators are highly stressed, as they must cope with a high cost of living, inadequate facilities, heavy 
teaching loads, inadequate training for the teaching of exceptional students, and an absence of administrative support (Hamann, \& Gordon, 2000). However, teachers in MDCPS must also cope with a large “culturally/linguistically diverse” population of émigré students. This is evidenced by the percentage of English Language Learners in M-DCPS (52.5\%) compared to the national average (8.5\%). Moreover, M-DCPS’s minority student population surpasses all other districts in Florida (Gudwin \& Salazar-Wallace, 2010).

\section{Research Questions}

Specific questions to be answered were:

1. Are there major stressors unique to elementary school music educators in MDCPS?

2. Do elementary school music educators in M-DCPS perceive their administrators as supporting music programs?

3. Has the accountability movement affected music programs and/or given music educators additional responsibilities that are a burden to music programs?

\section{Delimitations Regarding M-DCPS Elementary Music Educators and Attrition}

1. This study was limited to elementary music educators employed by M-DCPS. Therefore, educators who had left the field were not included.

\section{CHAPTER II}

\section{LITERATURE REVIEW}

The term "burn-out" can be defined as a state where a professional feels “exhaustion, depersonalization, a sense of reduced personal accomplishment, chronic fatigue, depression, and a desire to withdraw” (Dworkin, 1987, p. 25). It was first used in this manner in 1974 by Freudenberger, who changed the meaning of the word from the 
colloquial speech of the 1960s, when the term "burn-out” referenced drug abuse (Farber, 1983). The syndrome of burn-out is common among professionals who work with people in various capacities (Maslach, 1982). The concept of burn-out is difficult to define however, as there are many different causes, manifestations, and symptoms (Farber, 1983). A teacher's "idealism and excitement" in their preliminary years of teaching can be a precursor for the condition of burn-out (Gudwin \& Salazar-Wallace, 2010). Feelings that abound when one is undergoing burn-out include "helplessness, hopelessness, and entrapment” (Maslach \& Pines, 1988; Cedoline 1982). Burn-out is costly for school districts because it decreases productivity and necessitates replacing experienced educators (for whom the district has invested time and training) with less experienced educators (Pines \& Aronson, 1988; Blazer, 2010). Additionally, professionals who experience burn-out tend to be absent from work and to suffer from emotional and physical health problems more often than those who do not (Dworkin, 1987). Environment vs. Individual Personality

Maslach and Pines (1988) raised an important question as to whether burn-out is a result of individual personality or the work-environment. This idea is similar to the psychological concept of nature or nurture; they state however, that it would not be possible to create an experiment to differentiate between these two theories because of "ethical concerns." These two factors speak to the core of burn-out and the reactions to "mismatches" posed by an environment for individuals, and how these reactions can differ due to innumerable and unique work situations created by an institution. There are countless levels of individual "perceptions, skills, tolerance for job pressure, and vulnerability to dysfunctional outcomes," and these are the key modifiers of the stress- 
strain relationship (Guglielmi \& Tatrow, 1998, p.63). After 25 years of research into these two factors however, Maslach and Pines (1988) assert that the work environment has constantly surpassed the individual as a dependent factor in burn-out, as any human being will eventually burn-out given the proper environmental conditions. Additionally, burn-out is not caused by a single situation or problem; it is caused by a progression of issues (Farber, 1983).

Major issues in teaching have been identified by Maslach and Leiter (1997) as "work-overload (or under load in some cases), lack of control over policies, insufficient reward, unfairness in the system, and value conflict” (p. 26). As caring professionals, teachers cope with these situations as best they can. To handle work-overload they might take work home or stay extra hours, but this may have a negative impact on a teacher’s work/life balance. Little can be done regarding local or federal government policies, such as the recently enacted class size amendment. These policies, coupled with a general feeling of "lack of autonomy" in the school, can make teachers more prone to burn-out (Blazer, 2010). Salary too has been a concern for educators, as its performance has traditionally been substandard in keeping up with the cost of living. Job security and a feeling of "self-efficacy" can be more important however, unless money is tied to "recognition,” such as when bonuses are granted due to high FCAT scores (Pines \& Aronson, 1988, p.38).

In large bureaucratic public school systems there are bound to be circumstances that are deemed unfair. These unfair circumstances may produce feelings of distrust within organizations as they imply that those in charge do not respect music educators as professionals. These feelings may appear most intense during evaluations, as this is 
where teachers are judged on their job performance by administrators. However, "day to day interactions can produce these feelings that intensify burn-out, especially when there are incongruities between pay and job responsibilities” (Maslach \& Leiter, p. 15).

\section{Music Teachers and Burn-out}

It stands to reason that elementary music teachers would be prone to work related stress. There are many extra-curricular activities that fall under the responsibility of the music teacher, such as after school rehearsals, holiday shows, large numbers of students in music classes, and teaching in multiple schools (Allsup, 2005). Many parents, teachers, and administrators only see the finished product of these activities. They do not have an opportunity to experience what a music teacher goes through to get to a finished product. Some additional work responsibilities include early/late rehearsals, trips on the weekend, constant parent-teacher communication/conferences, professional development, and lesson preparation (Hamann \& Gordon, 2000). Many teachers must also perform extra duties not related to teaching music, such as supervision of students or reading tutoring, which means extra responsibilities for the music teacher. These additional roles can create an environment where teaching effectively is impossible because there may not be enough time or suitable resources to accomplish all that is required (Scheib, 2003).

\section{CHAPTER III \\ METHODOLOGY}

The Maslach Burn-out Inventory (M.B.I.) has been the prevailing instrument for the assessment of burn-out in human services careers since the 1970s. I developed a modified M.B.I. to be used in the context of elementary music education. This study 
consisted of five parts: an initial paper questionnaire, data analysis, subject selection, a final e-mailed questionnaire, and further data analysis.

\section{Description of the Participants}

This study used two representative sample groups. One group was selected from attendees at the FMENC in Tampa who had completed a survey questionnaire. The second representative sample consisted of randomly selected M-DCPS general music educators who were invited to take the same survey online. Completed questionnaires were sorted according to whether they were completed by M-DCPS teachers or teachers employed by various other counties throughout Florida. The questionnaires were coded with numbers, in order to maintain confidentiality.

\section{The M-DCPS Sample (Sample One)}

Sample one drew from music educators employed at 50 randomly selected schools. Subject selection consisted of educators chosen via a random number generator of M-DCPS mail location codes. These subjects were sent an e-mail (Appendix B) with the survey link (http://www.surveymonkey.com/s/WX3T8MN). This process ensured a direct connection to a secure site on which teachers could participate in the study. Subjects were informed, via an attached consent form, that the survey would be completely anonymous. Moreover, the first survey question was: "I agree to participate in this survey and understand that I can close it at any time without any consequences.” Questions about overall demographics were included in order to identify important commonalities. 13 questionnaires were obtained via the SurveyMonkey website. The online survey (Appendix C) contained a required extra question related to informed consent. All participants who completed the questionnaire at the FMENC were given a 
verbal consent, thus the extra question was not required. The majority of valid M-DCPS questionnaires (21) were collected at the FMENC. Questionnaires completed by MDCPS teachers who attended the conference were included in the M-DCPS sample.

Before e-mailing requests to complete the online survey, a permission request was sent to principals of the 50 randomly selected M-DCPS schools (Appendix A), as required by the M-DCPS Research Review Committee (M-DCPS RRC). The school selection process involved a random number generator to select participants by school number through the M-DCPS district e-mail. The M-DCPS RRC approval letter was also sent to the 50 principals to expedite the individual school principal approval process. Three principals refused to allow participation in this study. One refused because the school's teacher had just resigned to pursue another field. A second refused because their music teacher had been placed in a classroom teacher position. The third principal simply did not desire for that music teacher be contacted.

Other Florida Counties Sample (Sample Two)

For sample two, permission to set up a display at the FMENC in Tampa was sought from and granted by convention organizers. I was permitted to display my project and solicit participants at the conference curriculum fair. The fair was located in close proximity to the university booths in the convention hall. Data collection consisted of paper questionnaires distributed to and completed by Florida music educators who attended the FMENC. Sample two participants were teachers from many different Florida counties, including Alachua, Brevard, Broward, Columbia, Duval, Hernando, Hillsborough, Jackson, Jefferson, Lee, Leon, Manatee, Okeechobee, Orange, Osceola, Palm Beach, Pinellas, Polk, Seminole, St. Johns, St. Lucie, Sumter, Volusia, and Walton. 


\section{The Survey Instrument}

The teacher burn-out survey was designed in order to rate the role of 20 factors related to elementary music teacher burn-out. A Likert rating scale of one to six was established for each item, six being the "other” category for which subjects could write/type in a response. Participants were asked to check off their level of agreement with statements related to their careers. Ten general demographic questions were asked at the end of the questionnaire. In addition, two fill-in-the-blank questions asking for additional comments were used at the end of the survey to collect qualitative data. Actual survey questions can be seen in the following table.

\section{TABLE I: The Survey}

1. There are certain aspects related to my schools preparation for the Florida Comprehensive Assessment Test (FCAT) that take away from my program (e.g., student pull out, planning time being taken for FCAT activities, intervention I must teach, etc.).

2. I feel that my school's administration supports/values my program.

3. I often have difficulty facing another workday when I wake up in the morning.

4. I spend time at my school performing non-music related duties (e.g., reading tutoring, supervision duty, etc.).

5. My job has made me unsympathetic towards others.

6. I feel physically drained at the end of the workday.

7. I have too much of a workload on a weekly basis.

8. I feel that I have a balance between work and my home life.

9. I find myself not wanting to see certain classes/or students.

10. I feel totally emotionally drained at the end of the workday. 
11. My work is affecting my health (headaches, high blood pressure, insomnia, etc.).

12. I feel that I am very isolated at work, and cannot confide in others.

13. The politics of my school hinder me at work.

14. I feel that I am burned out as a teacher.

15. There are times when I am frustrated by trying to reach students whose native language is not English.

16. I find myself emotional exhausted on a weekly basis.

17. What is your age range?

18. Do you teach at more than one school?

19. Do you have your own classroom?

20. What is your current relationship status?

21. What county do you work in?

22. What is your major area of expertise?

23. What is your gender?

24. How long have you been teaching?

25. Do you have school-aged children?

26. What is your highest level of education?

27. What is the most stressful area of your life right now?

28. Do you have any additional comments about your job as a music educator and your level of stress?

\section{Data Analysis}

Data gained from these questionnaires determined the most significant causes of burn-out via a comparison of burn-out scores calculated for each participant. These data 
were thoroughly examined using descriptive statistical methods and a Mann-Whitney U (non-parametric test). There were four items that showed a difference in ranks according to Z scores on the Mann-Whitney U between these two populations. In order of significance these were: What is your gender? What is your age range? How long have you been teaching? I find myself not wanting to see certain classes/or students. These results will further be described and discussed in Chapter Four. However, significant Z scores and P values have been highlighted in bold on Table II, which follows:

TABLE II: Test Statistics (Mann-Whitney U/Wilcoxon W)

\begin{tabular}{|l|r|r|r|r|}
\hline & $\begin{array}{c}\text { Mann- } \\
\text { Whitney U }\end{array}$ & $\begin{array}{c}\text { Wilcoxon } \\
\text { W }\end{array}$ & Z & $\begin{array}{c}\text { Asymp. } \\
\text { Sig. (2- } \\
\text { tailed) }\end{array}$ \\
\hline $\begin{array}{l}\text { I understand that this survey is totally voluntary } \\
\text { and I can stop taking at any time if I choose. Do } \\
\text { you consent to taking this survey? }\end{array}$ & 748.000 & 1738.000 & .000 & 1.000 \\
\hline $\begin{array}{l}\text { There are certain aspects related to my schools } \\
\text { preparation for the Florida Comprehensive } \\
\text { Assessment Test (FCAT) that take away from my } \\
\text { program (e.g., student pull out, planning time being } \\
\text { taken for FCAT activities, intervention I must } \\
\text { teach, etc.). }\end{array}$ & 593.500 & 1154.500 & -1.716 & .086 \\
\hline $\begin{array}{l}\text { I feel that my school's administration } \\
\text { supports/values my program. }\end{array}$ & 694.000 & 1684.000 & -.586 & .558 \\
\hline $\begin{array}{l}\text { I have difficulty facing another workday when I } \\
\text { wake up in the morning. }\end{array}$ & 702.000 & 1297.000 & -.494 & .622 \\
\hline $\begin{array}{l}\text { I spend time at my school performing non-music } \\
\text { related duties (e.g., reading tutoring, supervision } \\
\text { duty, etc.). }\end{array}$ & 569.500 & 1559.500 & -1.474 & .141 \\
\hline $\begin{array}{l}\text { My job has made me unsympathetic towards } \\
\text { others. }\end{array}$ & 536.000 & 1482.000 & -1.958 & .05017 \\
\hline I feel physically drained at the end of the workday. & 736.500 & 1726.500 & -.124 & .901 \\
\hline $\begin{array}{l}\text { I feel that I have a balance between work and my } \\
\text { home life. }\end{array}$ & 722.000 & 1668.000 & -.096 & .924 \\
\hline
\end{tabular}




\begin{tabular}{|l|r|r|r|r|}
\hline $\begin{array}{l}\text { I feel totally emotionally drained at the end of the } \\
\text { workday. }\end{array}$ & 656.500 & 1251.500 & -.952 & .341 \\
\hline $\begin{array}{l}\text { My work is affecting my health (headaches, high } \\
\text { blood pressure, insomnia, etc. . .). }\end{array}$ & 665.000 & 1655.000 & -.865 & .387 \\
\hline $\begin{array}{l}\text { I feel that I am very isolated at work, and cannot } \\
\text { confide in others. }\end{array}$ & 582.000 & 1572.000 & -1.528 & .127 \\
\hline $\begin{array}{l}\text { The politics of my school have hindered me at } \\
\text { work. }\end{array}$ & 681.500 & 1276.500 & -.689 & .491 \\
\hline I feel that I am burned out as a teacher. & 699.000 & 1294.000 & -.514 & .607 \\
\hline I have too much of a workload on a weekly basis. & 716.500 & 1311.500 & -.332 & .740 \\
\hline $\begin{array}{l}\text { There are times when I am frustrated by trying to } \\
\text { reach students who home language is not English. }\end{array}$ & 703.000 & 1693.000 & -.464 & .642 \\
\hline $\begin{array}{l}\text { I find myself emotional exhausted on a weekly } \\
\text { basis. }\end{array}$ & 747.000 & 1737.000 & -.011 & .992 \\
\hline Do you have school-aged children? & 627.000 & 1617.000 & -1.342 & .180 \\
\hline What is your highest level of education? & 583.000 & 1573.000 & -1.860 & .063 \\
\hline What is your age range? & 430.000 & 1376.000 & $-\mathbf{- 3 . 2 4 8}$ & $\mathbf{. 0 0 1}$ \\
\hline Do you have your own classroom? & 743.000 & 1733.000 & -.073 & .942 \\
\hline Do you teach at more than one school? & 701.000 & 1296.000 & -.786 & .432 \\
\hline What is your current relationship status? & 628.000 & 1531.000 & -.586 & .558 \\
\hline What is your major area of expertise? & 489.000 & 924.000 & -1.025 & .306 \\
\hline What is your gender? & 442.500 & 1037.500 & $-\mathbf{- 4 . 1 1 9}$ & $\mathbf{. 0 0 0}$ \\
\hline How long have you been teaching? & 509.500 & 1104.500 & $\mathbf{- 2 . 5 6 4}$ & $\mathbf{. 0 1 0}$ \\
\hline $\begin{array}{l}\text { I find myself not wanting to see certain } \\
\text { classes/or students. }\end{array}$ & 733.000 & 1723.000 & -.151 & .880 \\
\hline Burn-out Total Score & & & & \\
\hline
\end{tabular}

\section{CHAPTER IV}

\section{RESULTS}

There were 98 questionnaires filled out by Florida elementary music teachers, 34 from M-DCPS music teachers (13 of these were obtained via SurveyMonkey, and while this was only a 26 percent return rate, this deficit was made up for at the FMENC) and 54 from music teachers employed in other counties. Ten of these questionnaires contained 
too many unanswered items to be useable for the study. Thus the total comparative statistics were taken from 34 valid surveys for M-DCPS and 44 valid surveys for the other county sample for a total of 78 participants.

\section{A Comparison of Percentages}

The following tables are graphical representatives of the comparison data retrieved from the survey samples. For purposes of clarity, "agree/somewhat agree will be referred to as a "positive response”, neither agree nor disagree will be referred to as a "neutral response”, and somewhat disagree, or disagree will be referred to as a "negative response.”

TABLE III: Comparative Frequencies for Samples One and Two

Question 1: There are certain aspects related to my school's preparation for the Florida Comprehensive Assessment Test (FCAT) that take away from my program (e.g. student pull-out, planning time being taken for FCAT activities, intervention I must teach, etc.)

\begin{tabular}{|c|c|c|c|c|c|c|}
\hline County & & & Frequency & Percent & $\begin{array}{l}\text { Valid } \\
\text { Percent }\end{array}$ & $\begin{array}{l}\text { Cumulative } \\
\text { Percent }\end{array}$ \\
\hline \multirow{8}{*}{$\begin{array}{l}\text { Sample } \\
\text { One }\end{array}$} & Valid & 1 Agree & 27 & 79.4 & 81.8 & 81.8 \\
\hline & & 2 Somewhat Agree & 3 & 8.8 & 9.1 & 90.9 \\
\hline & & $\begin{array}{l}3 \text { Neither Agree nor } \\
\text { Disagree }\end{array}$ & 2 & 5.9 & 6.1 & 97.0 \\
\hline & & $\begin{array}{l}4 \text { Somewhat } \\
\text { Disagree }\end{array}$ & & & & \\
\hline & & 5 Disagree & 1 & 2.9 & 3.0 & 100.0 \\
\hline & & Total & 33 & 97.1 & 100.00 & \\
\hline & Missing & System & 1 & 2.9 & & \\
\hline & \multicolumn{2}{|l|}{ Total } & 34 & 100.0 & & \\
\hline \multirow{6}{*}{$\begin{array}{l}\text { Sample } \\
\text { Two }\end{array}$} & Valid & 1 Agree & 28 & 63.6 & 63.6 & 63.6 \\
\hline & & 2 Somewhat Agree & 9 & 20.5 & 20.5 & 84.1 \\
\hline & & $\begin{array}{l}3 \text { Neither Agree nor } \\
\text { Disagree }\end{array}$ & 1 & 2.3 & 2.63 & 86.4 \\
\hline & & $\begin{array}{l}4 \text { Somewhat } \\
\text { Disagree }\end{array}$ & 4 & 9.1 & 9.1 & 95.5 \\
\hline & & 5 Disagree & 2 & 4.5 & 4.5 & 100.0 \\
\hline & \multicolumn{2}{|l|}{ Total } & 44 & 100.0 & 100.0 & \\
\hline
\end{tabular}

The responses to this question were the closest to achieving statistically significant levels of difference. Percentages for a positive response to this item for sample one were an 
overwhelming $90.9 \%$, compared to $84.1 \%$ for sample two. Combined, these percentages reached a rate of $88.5 \%$.

Question 2: I feel that my school's administration supports/values my program.

\begin{tabular}{|c|c|c|c|c|c|c|}
\hline County & & & Frequency & Percent & $\begin{array}{l}\text { Valid } \\
\text { Percent }\end{array}$ & $\begin{array}{l}\text { Cumulative } \\
\text { Percent }\end{array}$ \\
\hline \multirow{6}{*}{$\begin{array}{l}\text { Sample } \\
\text { One }\end{array}$} & Valid & 1 Agree & 16 & 47.1 & 47.1 & 47.1 \\
\hline & & 2 Somewhat Agree & 8 & 23.5 & 23.5 & 70.6 \\
\hline & & $\begin{array}{l}3 \text { Neither Agree } \\
\text { nor Disagree }\end{array}$ & 2 & 5.9 & 5.9 & 76.5 \\
\hline & & $\begin{array}{l}4 \text { Somewhat } \\
\text { Disagree }\end{array}$ & 4 & 11.8 & 11.8 & 88.2 \\
\hline & & 5 Disagree & 4 & 11.8 & 11.8 & 100.0 \\
\hline & Total & & 34 & 100.0 & 100.0 & \\
\hline \multirow{6}{*}{$\begin{array}{l}\text { Sample } \\
\text { Two } \\
\end{array}$} & Valid & 1 Agree & 19 & 43.2 & 43.2 & 43.2 \\
\hline & & 2 Somewhat Agree & 20 & 45.5 & 45.5 & 88.6 \\
\hline & & $\begin{array}{l}3 \text { Neither Agree } \\
\text { nor Disagree }\end{array}$ & 2 & 4.5 & 4.5 & 93.2 \\
\hline & & $\begin{array}{l}4 \text { Somewhat } \\
\text { Disagree }\end{array}$ & 1 & 2.3 & 2.3 & 95.5 \\
\hline & & 5 Disagree & 2 & 4.5 & 4.5 & 100.0 \\
\hline & Total & & 44 & 100.0 & 100.0 & \\
\hline
\end{tabular}

Combined positive responses to this question reached $70.6 \%$ for sample one and 87.7 for sample two.

Question 3: I have difficulty facing another day when I wake up in the morning.

\begin{tabular}{|c|c|c|c|c|c|c|}
\hline County & & & Frequency & Percent & $\begin{array}{l}\text { Valid } \\
\text { Percent }\end{array}$ & $\begin{array}{l}\text { Cumulative } \\
\text { Percent }\end{array}$ \\
\hline \multirow{6}{*}{$\begin{array}{l}\text { Sample } \\
\text { One }\end{array}$} & Valid & 1 Agree & 4 & 11.8 & 11.8 & 11.8 \\
\hline & & 2 Somewhat Agree & 7 & 20.6 & 20.6 & 32.4 \\
\hline & & $\begin{array}{l}3 \text { Neither Agree nor } \\
\text { Disagree }\end{array}$ & 4 & 11.8 & 11.8 & 44.1 \\
\hline & & $\begin{array}{l}4 \text { Somewhat } \\
\text { Disagree }\end{array}$ & 3 & 8.8 & 8.8 & 52.9 \\
\hline & & 5 Disagree & 16 & 47.1 & 47.1 & 100.0 \\
\hline & Total & & 34 & 100.0 & 100.0 & \\
\hline \multirow{6}{*}{$\begin{array}{l}\text { Sample } \\
\text { Two }\end{array}$} & Valid & 1 Agree & 4 & 9.1 & 9.1 & 9.1 \\
\hline & & 2 Somewhat Agree & 7 & 15.9 & 15.9 & 25.0 \\
\hline & & $\begin{array}{l}3 \text { Neither Agree nor } \\
\text { Disagree }\end{array}$ & 3 & 6.8 & 6.8 & 31.8 \\
\hline & & $\begin{array}{l}4 \text { Somewhat } \\
\text { Disagree }\end{array}$ & 9 & 20.5 & 20.5 & 52.3 \\
\hline & & 5 Disagree & 21 & 47.7 & 47.7 & 100.0 \\
\hline & Total & & 44 & 100.0 & 100.0 & \\
\hline
\end{tabular}


Combined positive responses: were skewed more toward the negative on this question, only $32.4 \%$ of sample one had positive response, compared to a $25 \%$ positive response from sample two. While these numbers do represent almost a third of participants $(30.7 \%$ of all participants), there were $61.4 \%$ of total participants that had a negative response to this item.

Question 4: I spend time at my school performing non-music related duties (e.g., reading tutoring, supervision duty, etc.).

\begin{tabular}{|c|c|c|c|c|c|c|}
\hline County & & & Frequency & Percent & $\begin{array}{l}\text { Valid } \\
\text { Percent }\end{array}$ & $\begin{array}{l}\text { Cumulative } \\
\text { Percent }\end{array}$ \\
\hline \multirow{8}{*}{$\begin{array}{l}\text { Sample } \\
\text { One }\end{array}$} & Valid & 1 Agree & 9 & 26.5 & 28.1 & 28.1 \\
\hline & & 2 Somewhat Agree & 9 & 26.5 & 28.1 & 56.3 \\
\hline & & $\begin{array}{l}3 \text { Neither Agree nor } \\
\text { Disagree }\end{array}$ & 1 & 2.9 & 3.1 & 59.4 \\
\hline & & $\begin{array}{l}4 \text { Somewhat } \\
\text { Disagree }\end{array}$ & 3 & 8.8 & 9.4 & 68.8 \\
\hline & & 5 Disagree & 10 & 29.4 & 31.3 & 100.0 \\
\hline & & Total & 32 & 94.1 & 100.0 & \\
\hline & Missing & System & 2 & 5.9 & & \\
\hline & Total & & 34 & 100.0 & & \\
\hline \multirow{6}{*}{$\begin{array}{l}\text { Sample } \\
\text { Two }\end{array}$} & Valid & 1 Agree & 18 & 40.9 & 40.9 & 40.9 \\
\hline & & 2 Somewhat Agree & 12 & 27.3 & 27.3 & 68.2 \\
\hline & & $\begin{array}{l}3 \text { Neither Agree nor } \\
\text { Disagree }\end{array}$ & 3 & 6.8 & 6.8 & 75.0 \\
\hline & & $\begin{array}{l}4 \text { Somewhat } \\
\text { Disagree }\end{array}$ & 4 & 9.1 & 9.1 & 84.1 \\
\hline & & 5 Disagree & 7 & 15.9 & 15.9 & 100.0 \\
\hline & Total & & 44 & 100.0 & 100.0 & \\
\hline
\end{tabular}

$56.2 \%$ of sample one and $68.2 \%$ of sample two had a positive response. The total percentage of all participants was surprising, at $64.7 \%$. 
Question 5: My job has made me unsympathetic towards others.

\begin{tabular}{|c|c|c|c|c|c|c|}
\hline County & & & Frequency & Percent & $\begin{array}{l}\text { Valid } \\
\text { Percent }\end{array}$ & $\begin{array}{l}\text { Cumulative } \\
\text { Percent }\end{array}$ \\
\hline \multirow{8}{*}{$\begin{array}{l}\text { Sample } \\
\text { One }\end{array}$} & Valid & 1 Agree & & & & \\
\hline & & 2 Somewhat Agree & 3 & 8.8 & 9.1 & 9.1 \\
\hline & & $\begin{array}{l}3 \text { Neither Agree nor } \\
\text { Disagree }\end{array}$ & 4 & 11.8 & 12.1 & 21.2 \\
\hline & & $\begin{array}{l}\text { 4 Somewhat } \\
\text { Disagree }\end{array}$ & 6 & 17.6 & 18.2 & 39.4 \\
\hline & & 5 Disagree & 20 & 58.8 & 60.6 & 100.0 \\
\hline & & Total & 33 & 91.1 & 100.0 & \\
\hline & Missing & System & 1 & 2.9 & & \\
\hline & Total & & 34 & 100.0 & & \\
\hline \multirow{8}{*}{$\begin{array}{l}\text { Sample } \\
\text { Two }\end{array}$} & Valid & 1 Agree & 1 & 2.3 & 2.3 & 2.3 \\
\hline & & 2 Somewhat Agree & 8 & 18.2 & 18.6 & 20.9 \\
\hline & & $\begin{array}{l}3 \text { Neither Agree nor } \\
\text { Disagree }\end{array}$ & 10 & 22.7 & 23.3 & 44.2 \\
\hline & & $\begin{array}{l}4 \text { Somewhat } \\
\text { Disagree }\end{array}$ & 6 & 13.6 & 14.0 & 58.1 \\
\hline & & 5 Disagree & 18 & 40.9 & 41.9 & 100.0 \\
\hline & & Total & 43 & 97.7 & 100.0 & \\
\hline & Missing & System & 1 & 2.3 & & \\
\hline & Total & & 44 & 100.0 & & \\
\hline
\end{tabular}

This item elicited a negative response for most respondents, only $16.5 \%$ of total participants had a positive response. Sample one has $21.2 \%$ and $20.9 \%$ for sample two. 
Question 6: I feel physically drained at the end of the workday.

\begin{tabular}{|c|c|c|c|c|c|c|}
\hline County & & & Frequency & Percent & $\begin{array}{l}\text { Valid } \\
\text { Percent }\end{array}$ & $\begin{array}{l}\text { Cumulative } \\
\text { Percent }\end{array}$ \\
\hline \multirow{6}{*}{$\begin{array}{l}\text { Sample } \\
\text { One }\end{array}$} & Valid & 1 Agree & 15 & 44.1 & 44.1 & 44.1 \\
\hline & & 2 Somewhat Agree & 11 & 32.4 & 32.4 & 76.5 \\
\hline & & $\begin{array}{l}3 \text { Neither Agree nor } \\
\text { Disagree }\end{array}$ & 2 & 5.9 & 5.9 & 82.4 \\
\hline & & $\begin{array}{l}\text { 4 Somewhat } \\
\text { Disagree }\end{array}$ & 3 & 8.8 & 8.8 & 91.2 \\
\hline & & 5 Disagree & 3 & 8.8 & 8.8 & 100.0 \\
\hline & Total & & 34 & 100.0 & 100.0 & \\
\hline \multirow{6}{*}{$\begin{array}{l}\text { Sample } \\
\text { Two }\end{array}$} & Valid & 1 Agree & 17 & 38.6 & 38.6 & 38.6 \\
\hline & & 2 Somewhat Agree & 20 & 45.5 & 45.5 & 84.1 \\
\hline & & $\begin{array}{l}3 \text { Neither Agree nor } \\
\text { Disagree }\end{array}$ & 3 & 6.8 & 6.8 & 90.9 \\
\hline & & $\begin{array}{l}4 \text { Somewhat } \\
\text { Disagree }\end{array}$ & 4 & 9.1 & 9.1 & 100.0 \\
\hline & & 5 Disagree & & & & \\
\hline & Total & & 44 & 100.0 & 100.0 & \\
\hline
\end{tabular}

This item was significant for both populations; the total percentage of participants that gave a positive response to this item was $79.3 \%$. For sample one this was a $76.5 \%$ and for sample two it was $84.1 \%$, a difference of $7.6 \%$.

Question 7: I have too much of a workload on a weekly basis.

\begin{tabular}{|c|c|c|c|c|c|c|}
\hline County & & & Frequency & Percent & $\begin{array}{l}\text { Valid } \\
\text { Percent }\end{array}$ & $\begin{array}{l}\text { Cumulative } \\
\text { Percent }\end{array}$ \\
\hline \multirow{6}{*}{$\begin{array}{l}\text { Sample } \\
\text { One }\end{array}$} & Valid & 1 Agree & 5 & 14.7 & 14.7 & 14.7 \\
\hline & & 2 Somewhat Agree & 16 & 47.1 & 47.1 & 61.8 \\
\hline & & $\begin{array}{l}3 \text { Neither Agree nor } \\
\text { Disagree }\end{array}$ & 8 & 23.5 & 23.5 & 85.3 \\
\hline & & $\begin{array}{l}\text { 4 Somewhat } \\
\text { Disagree }\end{array}$ & 1 & 2.9 & 2.9 & 88.2 \\
\hline & & 5 Disagree & 4 & 11.8 & 11.8 & 100.0 \\
\hline & Total & & 34 & 100.0 & 100.0 & \\
\hline \multirow{6}{*}{$\begin{array}{l}\text { Sample } \\
\text { Two }\end{array}$} & Valid & 1 Agree & 9 & 20.5 & 20.5 & 20.5 \\
\hline & & 2 Somewhat Agree & 16 & 36.4 & 36.4 & 56.8 \\
\hline & & $\begin{array}{l}3 \text { Neither Agree nor } \\
\text { Disagree }\end{array}$ & 7 & 15.9 & 15.9 & 72.7 \\
\hline & & $\begin{array}{l}4 \text { Somewhat } \\
\text { Disagree }\end{array}$ & 4 & 9.1 & 9.1 & 81.8 \\
\hline & & 5 Disagree & 8 & 18.2 & 18.2 & 100.0 \\
\hline & Total & & 44 & 100.0 & 100.0 & \\
\hline
\end{tabular}


This item was collectively similar across all participants, $61.8 \%$ of sample one, and $56.9 \%$ of sample two had a positive response. For the total number of participants this percentage was 59.8.

Question 8: I feel that I have a balance between work and my home life.

\begin{tabular}{|c|c|c|c|c|c|c|}
\hline County & & & Frequency & Percent & $\begin{array}{l}\text { Valid } \\
\text { Percent }\end{array}$ & $\begin{array}{l}\text { Cumulative } \\
\text { Percent }\end{array}$ \\
\hline \multirow{6}{*}{$\begin{array}{l}\text { Sample } \\
\text { One }\end{array}$} & Valid & 1 Agree & 10 & 29.4 & 29.4 & 29.4 \\
\hline & & 2 Somewhat Agree & 10 & 29.4 & 29.4 & 58.8 \\
\hline & & $\begin{array}{l}3 \text { Neither Agree nor } \\
\text { Disagree }\end{array}$ & 4 & 11.8 & 11.8 & 70.6 \\
\hline & & $\begin{array}{l}4 \text { Somewhat } \\
\text { Disagree }\end{array}$ & 6 & 17.6 & 17.6 & 88.2 \\
\hline & & 5 Disagree & 4 & 11.8 & 11.8 & 100.0 \\
\hline & Total & & 34 & 100.0 & 100.0 & \\
\hline \multirow{8}{*}{$\begin{array}{l}\text { Sample } \\
\text { Two }\end{array}$} & Valid & 1 Agree & 12 & 27.3 & 27.9 & 27.9 \\
\hline & & 2 Somewhat Agree & 15 & 34.1 & 34.9 & 62.8 \\
\hline & & $\begin{array}{l}3 \text { Neither Agree nor } \\
\text { Disagree }\end{array}$ & 2 & 4.5 & 4.7 & 67.4 \\
\hline & & $\begin{array}{l}4 \text { Somewhat } \\
\text { Disagree }\end{array}$ & 11 & 25.0 & 25.6 & 93.0 \\
\hline & & 5 Disagree & 3 & 6.8 & 7.0 & 100.0 \\
\hline & & Total & 43 & 97.7 & 100.0 & \\
\hline & Missing & System & 1 & 2.3 & & \\
\hline & Total & & 44 & 100.0 & & \\
\hline
\end{tabular}

The results of this item for both samples were comparable. 58.8\% for sample one, $61.4 \%$ for sample two, and $34.9 \%$ for the total sample. 
Question 9: I find myself not wanting to see certain classes/or students.

\begin{tabular}{|c|c|c|c|c|c|c|}
\hline County & & & Frequency & Percent & $\begin{array}{l}\text { Valid } \\
\text { Percent }\end{array}$ & $\begin{array}{l}\text { Cumulative } \\
\text { Percent }\end{array}$ \\
\hline \multirow{6}{*}{$\begin{array}{l}\text { Sample } \\
\text { One }\end{array}$} & Valid & 1 Agree & 19 & 55.9 & 55.9 & 55.9 \\
\hline & & 2 Somewhat Agree & 12 & 35.3 & 35.3 & 91.2 \\
\hline & & $\begin{array}{l}3 \text { Neither Agree nor } \\
\text { Disagree }\end{array}$ & 1 & 2.9 & 2.9 & 94.1 \\
\hline & & $\begin{array}{l}\text { 4 Somewhat } \\
\text { Disagree }\end{array}$ & 2 & 5.9 & 5.9 & 100.0 \\
\hline & & 5 Disagree & & & & \\
\hline & Total & & 34 & 100.0 & 100.0 & \\
\hline \multirow{6}{*}{$\begin{array}{l}\text { Sample } \\
\text { Two }\end{array}$} & Valid & 1 Agree & 15 & 34.1 & 34.1 & 34.1 \\
\hline & & 2 Somewhat Agree & 14 & 31.8 & 31.8 & 65.9 \\
\hline & & $\begin{array}{l}3 \text { Neither Agree nor } \\
\text { Disagree }\end{array}$ & 4 & 9.1 & 9.1 & 75.0 \\
\hline & & $\begin{array}{l}4 \text { Somewhat } \\
\text { Disagree }\end{array}$ & 6 & 13.6 & 13.6 & 88.6 \\
\hline & & 5 Disagree & 5 & 11.4 & 11.4 & 100.0 \\
\hline & Total & & 44 & 100.0 & 100.0 & \\
\hline
\end{tabular}

There was a $25.3 \%$ difference here between samples with a $91.2 \%$ for sample one, a $65.9 \%$ for sample two, and a $77 \%$ total for all participants. This was found to be statistically significant.

Question 10: I feel totally emotionally drained at the end of the workday.

\begin{tabular}{|c|c|c|c|c|c|c|}
\hline County & & & Frequency & Percent & $\begin{array}{l}\text { Valid } \\
\text { Percent }\end{array}$ & $\begin{array}{l}\text { Cumulative } \\
\text { Percent }\end{array}$ \\
\hline \multirow{6}{*}{$\begin{array}{l}\text { Sample } \\
\text { One }\end{array}$} & Valid & 1 Agree & 5 & 14.7 & 14.7 & 14.7 \\
\hline & & 2 Somewhat Agree & 14 & 41.2 & 41.2 & 55.9 \\
\hline & & $\begin{array}{l}3 \text { Neither Agree nor } \\
\text { Disagree }\end{array}$ & 5 & 14.7 & 14.7 & 70.6 \\
\hline & & $\begin{array}{l}\text { 4 Somewhat } \\
\text { Disagree }\end{array}$ & 4 & 11.8 & 11.8 & 82.4 \\
\hline & & 5 Disagree & 6 & 17.6 & 17.6 & 100.0 \\
\hline & Total & & 34 & 100.0 & 100.0 & \\
\hline \multirow{6}{*}{$\begin{array}{l}\text { Sample } \\
\text { Two }\end{array}$} & Valid & 1 Agree & 4 & 9.1 & 9.1 & 9.1 \\
\hline & & 2 Somewhat Agree & 12 & 27.3 & 27.3 & 36.4 \\
\hline & & $\begin{array}{l}3 \text { Neither Agree nor } \\
\text { Disagree }\end{array}$ & 15 & 34.1 & 34.1 & 70.5 \\
\hline & & $\begin{array}{l}4 \text { Somewhat } \\
\text { Disagree }\end{array}$ & 8 & 18.2 & 18.2 & 88.6 \\
\hline & & 5 Disagree & 5 & 11.4 & 11.4 & 100.0 \\
\hline & Total & & 44 & 100.0 & 100.0 & \\
\hline
\end{tabular}

Here again we see that sample one has a larger percentage at 55.9\%, compared to $36.4 \%$ for sample two (it's a difference of 19.5), and $43.6 \%$ the total population. 
Question 11: My work is affecting my health (headaches, high blood pressure, insomnia, etc.).

\begin{tabular}{|c|c|c|c|c|c|c|}
\hline County & & & Frequency & Percent & $\begin{array}{l}\text { Valid } \\
\text { Percent }\end{array}$ & $\begin{array}{l}\text { Cumulative } \\
\text { Percent }\end{array}$ \\
\hline \multirow{6}{*}{$\begin{array}{l}\text { Sample } \\
\text { One }\end{array}$} & Valid & 1 Agree & 4 & 11.8 & 11.8 & 11.8 \\
\hline & & 2 Somewhat Agree & 11 & 32.4 & 32.4 & 44.1 \\
\hline & & $\begin{array}{l}3 \text { Neither Agree nor } \\
\text { Disagree }\end{array}$ & 4 & 11.8 & 11.8 & 55.9 \\
\hline & & $\begin{array}{l}4 \text { Somewhat } \\
\text { Disagree }\end{array}$ & 2 & 5.9 & 5.9 & 61.8 \\
\hline & & 5 Disagree & 13 & 38.2 & 38.2 & 100.0 \\
\hline & Total & & 34 & 100.0 & 100.0 & \\
\hline \multirow{7}{*}{$\begin{array}{l}\text { Sample } \\
\text { Two } \\
\end{array}$} & Valid & 1 Agree & 5 & 11.4 & 11.4 & 11.4 \\
\hline & & 2 Somewhat Agree & 17 & 38.6 & 38.6 & 50.0 \\
\hline & & $\begin{array}{l}3 \text { Neither Agree nor } \\
\text { Disagree }\end{array}$ & 4 & 9.1 & 9.1 & 59.1 \\
\hline & & $\begin{array}{l}4 \text { Somewhat } \\
\text { Disagree }\end{array}$ & 11 & 25.0 & 25.0 & 84.1 \\
\hline & & 5 Disagree & 5 & 11.4 & 11.4 & 95.5 \\
\hline & & 6 Other & 2 & 4.5 & 4.5 & 100.0 \\
\hline & Total & & 44 & 100.0 & 100.0 & \\
\hline
\end{tabular}

These results were quite high for the total population, virtually half, $48 \%$ of participants self-reporting health issues; these percentages were $44.2 \%$ for sample one, and $50 \%$ for sample two. 
Question 12: I feel that I am very isolated at work, and cannot confide in others.

\begin{tabular}{|c|c|c|c|c|c|c|}
\hline County & & & Frequency & Percent & $\begin{array}{l}\text { Valid } \\
\text { Percent }\end{array}$ & $\begin{array}{l}\text { Cumulative } \\
\text { Percent }\end{array}$ \\
\hline \multirow{8}{*}{$\begin{array}{l}\text { Sample } \\
\text { One }\end{array}$} & Valid & 1 Agree & 5 & 14.7 & 15.2 & 15.2 \\
\hline & & 2 Somewhat Agree & 8 & 23.5 & 24.2 & 39.4 \\
\hline & & $\begin{array}{l}3 \text { Neither Agree nor } \\
\text { Disagree }\end{array}$ & 4 & 11.8 & 12.1 & 51.5 \\
\hline & & $\begin{array}{l}4 \text { Somewhat } \\
\text { Disagree }\end{array}$ & 2 & 5.9 & 6.1 & 57.6 \\
\hline & & 5 Disagree & 14 & 41.2 & 42.4 & 100.0 \\
\hline & & Total & 33 & 97.1 & 100.0 & \\
\hline & Missing & System & 1 & 2.9 & & \\
\hline & Total & & 34 & 100.0 & & \\
\hline \multirow{6}{*}{$\begin{array}{l}\text { Sample } \\
\text { Two }\end{array}$} & Valid & 1 Agree & 8 & 18.2 & 18.2 & 18.2 \\
\hline & & 2 Somewhat Agree & 15 & 34.1 & 34.1 & 52.3 \\
\hline & & $\begin{array}{l}3 \text { Neither Agree nor } \\
\text { Disagree }\end{array}$ & 5 & 11.4 & 11.4 & 63.6 \\
\hline & & $\begin{array}{l}4 \text { Somewhat } \\
\text { Disagree }\end{array}$ & 8 & 18.2 & 18.2 & 81.8 \\
\hline & & 5 Disagree & 8 & 18.2 & 18.2 & 100.0 \\
\hline & Total & & 44 & 100.0 & 100.0 & \\
\hline
\end{tabular}

This item was higher by $12.9 \%$ for the sample two which had a 52.3\%. 39.4\% of sample one had a positive response to this item, and a total of $45.4 \%$ had a positive response to this item. 
Question 13: The politics of my school have hindered me at work.

\begin{tabular}{|c|c|c|c|c|c|c|}
\hline County & & & Frequency & Percent & $\begin{array}{l}\text { Valid } \\
\text { Percent }\end{array}$ & $\begin{array}{l}\text { Cumulative } \\
\text { Percent }\end{array}$ \\
\hline \multirow[t]{6}{*}{$\begin{array}{l}\text { Sample } \\
\text { One }\end{array}$} & Valid & 1 Agree & 8 & 23.5 & 23.5 & 23.5 \\
\hline & & 2 Somewhat Agree & 11 & 32.4 & 32.4 & 55.9 \\
\hline & & $\begin{array}{l}3 \text { Neither Agree nor } \\
\text { Disagree }\end{array}$ & 6 & 17.6 & 17.6 & 73.5 \\
\hline & & $\begin{array}{l}\text { 4 Somewhat } \\
\text { Disagree }\end{array}$ & 2 & 5.9 & 5.9 & 79.4 \\
\hline & & 5 Disagree & 7 & 20.6 & 20.6 & 100.0 \\
\hline & Total & & 34 & 100.0 & 100.0 & \\
\hline \multirow{6}{*}{$\begin{array}{l}\text { Sample } \\
\text { Two }\end{array}$} & Valid & 1 Agree & 7 & 15.9 & 15.9 & 15.9 \\
\hline & & 2 Somewhat Agree & 14 & 31.8 & 31.8 & 47.7 \\
\hline & & $\begin{array}{l}3 \text { Neither Agree nor } \\
\text { Disagree }\end{array}$ & 8 & 18.2 & 18.2 & 65.9 \\
\hline & & $\begin{array}{l}4 \text { Somewhat } \\
\text { Disagree }\end{array}$ & 8 & 18.2 & 18.2 & 84.1 \\
\hline & & 5 Disagree & 7 & 15.9 & 15.9 & 100.0 \\
\hline & Total & & 44 & 100.0 & 100.0 & \\
\hline
\end{tabular}

More than half of sample one (55.9\%) agreed with this statement, but the percentages was also high for sample two at $47.7 \%$ s, with the total sample at $49.4 \%$.

Question 14: I feel that I am burned out as a teacher.

\begin{tabular}{|c|c|c|c|c|c|c|}
\hline County & & & Frequency & Percent & $\begin{array}{l}\text { Valid } \\
\text { Percent }\end{array}$ & $\begin{array}{l}\text { Cumulative } \\
\text { Percent }\end{array}$ \\
\hline \multirow{7}{*}{$\begin{array}{l}\text { Sample } \\
\text { One }\end{array}$} & Valid & 1 Agree & 2 & 5.9 & 5.9 & 5.9 \\
\hline & & 2 Somewhat Agree & 10 & 29.4 & 29.4 & 35.3 \\
\hline & & $\begin{array}{l}3 \text { Neither Agree nor } \\
\text { Disagree }\end{array}$ & 6 & 17.6 & 17.6 & 52.9 \\
\hline & & $\begin{array}{l}\text { 4 Somewhat } \\
\text { Disagree }\end{array}$ & 3 & 8.8 & 8.8 & 61.8 \\
\hline & & 5 Disagree & 12 & 35.3 & 35.3 & 97.1 \\
\hline & & 6 Other & 1 & 2.9 & 2.9 & 100.0 \\
\hline & Total & & 34 & 100.0 & 100.0 & \\
\hline \multirow{6}{*}{$\begin{array}{l}\text { Sample } \\
\text { Two }\end{array}$} & Valid & 1 Agree & 3 & 6.8 & 6.8 & 6.8 \\
\hline & & 2 Somewhat Agree & 8 & 18.2 & 18.2 & 25.0 \\
\hline & & $\begin{array}{l}3 \text { Neither Agree nor } \\
\text { Disagree }\end{array}$ & 9 & 20.5 & 20.5 & 45.5 \\
\hline & & $\begin{array}{l}4 \text { Somewhat } \\
\text { Disagree }\end{array}$ & 6 & 13.6 & 13.6 & 59.1 \\
\hline & & 5 Disagree & 18 & 40.9 & 40.9 & 100.0 \\
\hline & Total & & 44 & 100.0 & 100.0 & \\
\hline
\end{tabular}

This item was $10.3 \%$ higher for sample one at $35.3 \%$, compared to $25 \%$ for sample two, and $31 \%$ for the total population. 
Question 15: There are times when I am frustrated by trying to reach students whose native language is not English.

\begin{tabular}{|c|c|c|c|c|c|c|}
\hline County & & & Frequency & Percent & $\begin{array}{l}\text { Valid } \\
\text { Percent }\end{array}$ & $\begin{array}{l}\text { Cumulative } \\
\text { Percent }\end{array}$ \\
\hline \multirow{6}{*}{$\begin{array}{l}\text { Sample } \\
\text { One }\end{array}$} & Valid & 1 Agree & 6 & 17.6 & 17.6 & 17.6 \\
\hline & & 2 Somewhat Agree & 6 & 17.6 & 17.6 & 35.3 \\
\hline & & $\begin{array}{l}3 \text { Neither Agree nor } \\
\text { Disagree }\end{array}$ & 4 & 11.8 & 11.8 & 47.1 \\
\hline & & $\begin{array}{l}4 \text { Somewhat } \\
\text { Disagree }\end{array}$ & 8 & 23.5 & 23.5 & 70.6 \\
\hline & & 5 Disagree & 10 & 29.4 & 29.4 & 100.0 \\
\hline & Total & & 34 & 100.0 & 100.0 & \\
\hline \multirow{6}{*}{$\begin{array}{l}\text { Sample } \\
\text { Two } \\
\end{array}$} & Valid & 1 Agree & 6 & 13.6 & 13.6 & 13.6 \\
\hline & & 2 Somewhat Agree & 12 & 27.3 & 27.3 & 40.9 \\
\hline & & $\begin{array}{l}3 \text { Neither Agree nor } \\
\text { Disagree }\end{array}$ & 7 & 15.9 & 15.9 & 56.8 \\
\hline & & $\begin{array}{l}4 \text { Somewhat } \\
\text { Disagree }\end{array}$ & 8 & 18.2 & 18.2 & 75.0 \\
\hline & & 5 Disagree & 11 & 25.0 & 25.0 & 100.0 \\
\hline & Total & & 44 & 100.0 & 100.0 & \\
\hline
\end{tabular}

This item did not yield too much of a significance for sample one (35.2\%), sample two was slightly higher at $40.9 \%$, and $36.7 \%$ of the total chose a positive statement related to this item. 
Question 16: I find myself emotional exhausted on a weekly basis.

\begin{tabular}{|c|c|c|c|c|c|c|}
\hline County & & & Frequency & Percent & $\begin{array}{l}\text { Valid } \\
\text { Percent }\end{array}$ & $\begin{array}{l}\text { Cumulative } \\
\text { Percent }\end{array}$ \\
\hline \multirow{6}{*}{$\begin{array}{l}\text { Sample } \\
\text { One }\end{array}$} & Valid & 1 Agree & 9 & 26.5 & 26.5 & 26.5 \\
\hline & & 2 Somewhat Agree & 10 & 29.4 & 29.4 & 55.9 \\
\hline & & $\begin{array}{l}3 \text { Neither Agree nor } \\
\text { Disagree }\end{array}$ & 2 & 5.9 & 5.9 & 61.8 \\
\hline & & $\begin{array}{l}4 \text { Somewhat } \\
\text { Disagree }\end{array}$ & 5 & 14.7 & 14.7 & 76.5 \\
\hline & & 5 Disagree & 8 & 23.5 & 23.5 & 100.0 \\
\hline & Total & & 34 & 100.0 & 100.0 & \\
\hline \multirow[t]{6}{*}{$\begin{array}{l}\text { Sample } \\
\text { Two } \\
\end{array}$} & Valid & 1 Agree & 4 & 9.1 & 9.1 & 9.1 \\
\hline & & 2 Somewhat Agree & 22 & 50.0 & 50.0 & 59.1 \\
\hline & & $\begin{array}{l}3 \text { Neither Agree nor } \\
\text { Disagree }\end{array}$ & 8 & 18.2 & 18.2 & 77.3 \\
\hline & & $\begin{array}{l}4 \text { Somewhat } \\
\text { Disagree }\end{array}$ & 6 & 13.6 & 13.6 & 90.9 \\
\hline & & 5 Disagree & 4 & 9.1 & 9.1 & 100.0 \\
\hline & Total & & 44 & 100.0 & 100.0 & \\
\hline
\end{tabular}

This item was yielded similar results for all participants $55.9 \%$ for sample one, $59.1 \%$ for sample two, and $57.4 \%$ for the total population sampled. 
The following graphs are related to demographic information for both samples, they have been placed next to each other for comparison, but some of the quantities for the measurements are independent. This was because in some instances the distributions for sample 1 differed greatly from those for sample 2 .

What is your age range?

Figure I: Age Ranges

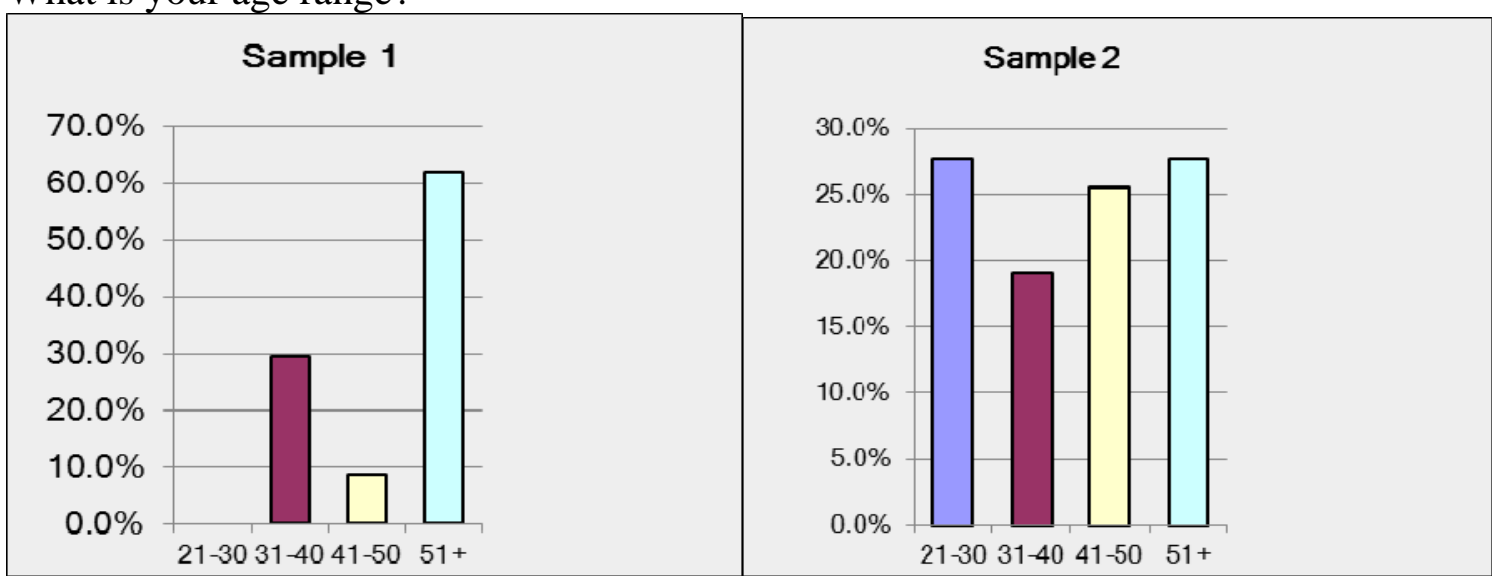

From these results we can see that sample one had a much higher percentage of teachers over the age of 51. Sample two had a much high percentage of younger teachers in the field.

Figure II: School Aged Children

Do you have school-aged children?

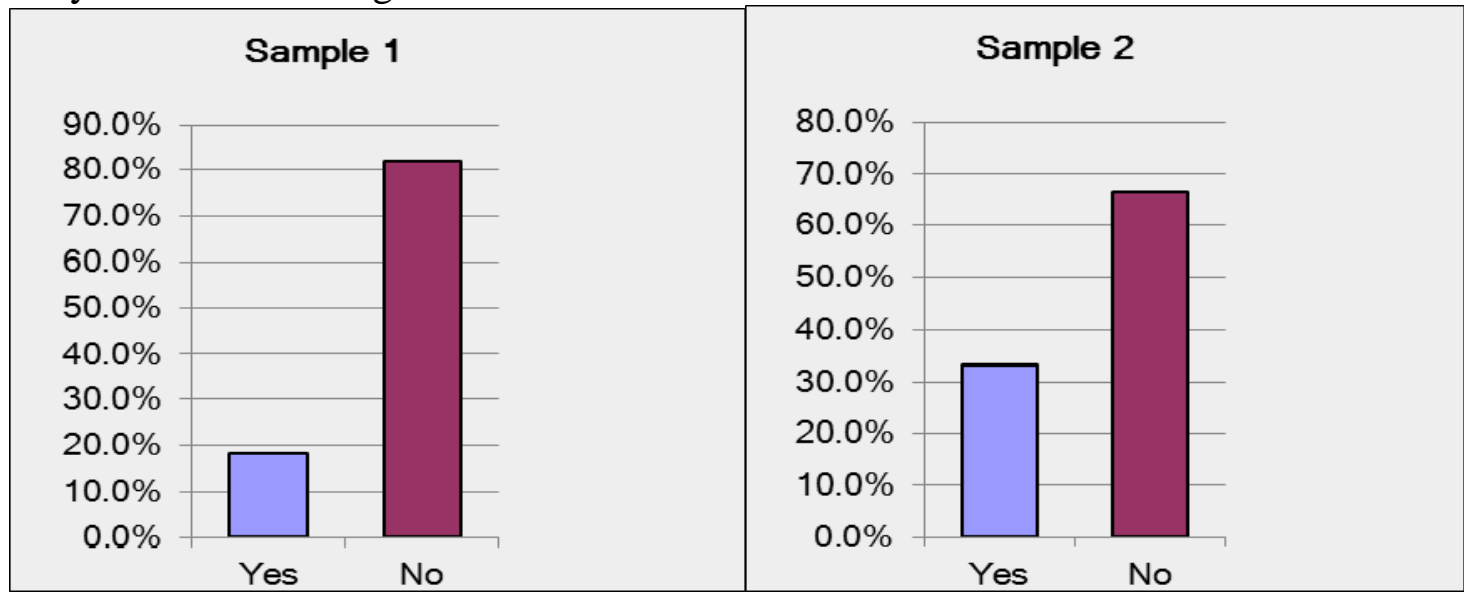

Sample two had a one-third higher percentage of school aged children. 
Figure III: Years of Experience

How long have you been teaching?

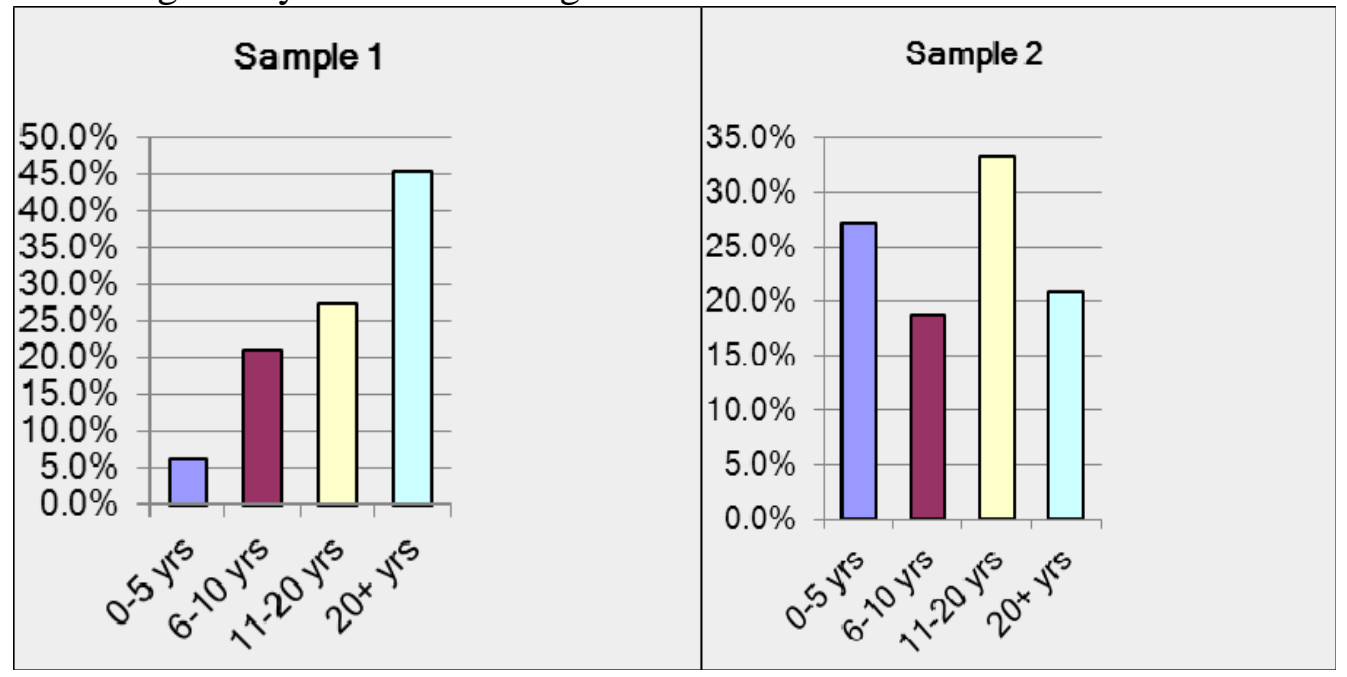

One can see that much like age range, sample 1 contained a much more experienced workforce compared to the more normally distributed results for sample 2.

Figure IV: Level of Education

What is your highest level of education?

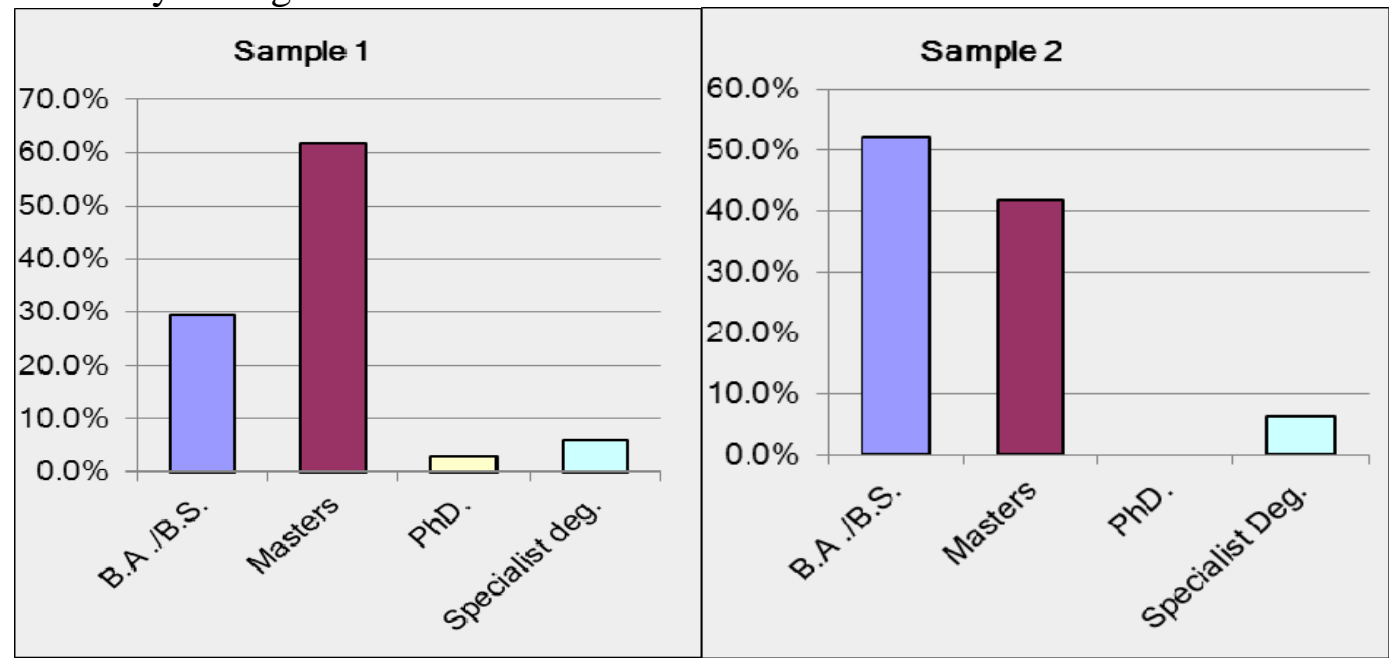

Overall almost half of the total sample had attained a master's degree, Education level: 33.3\% had a B.S. /B.A degree, 31\% had a master's degree, 3\% had a specialist degree, and $1 \%$ had received a doctorate. 
Figure III: Relationship Status

What is your current relationship status?

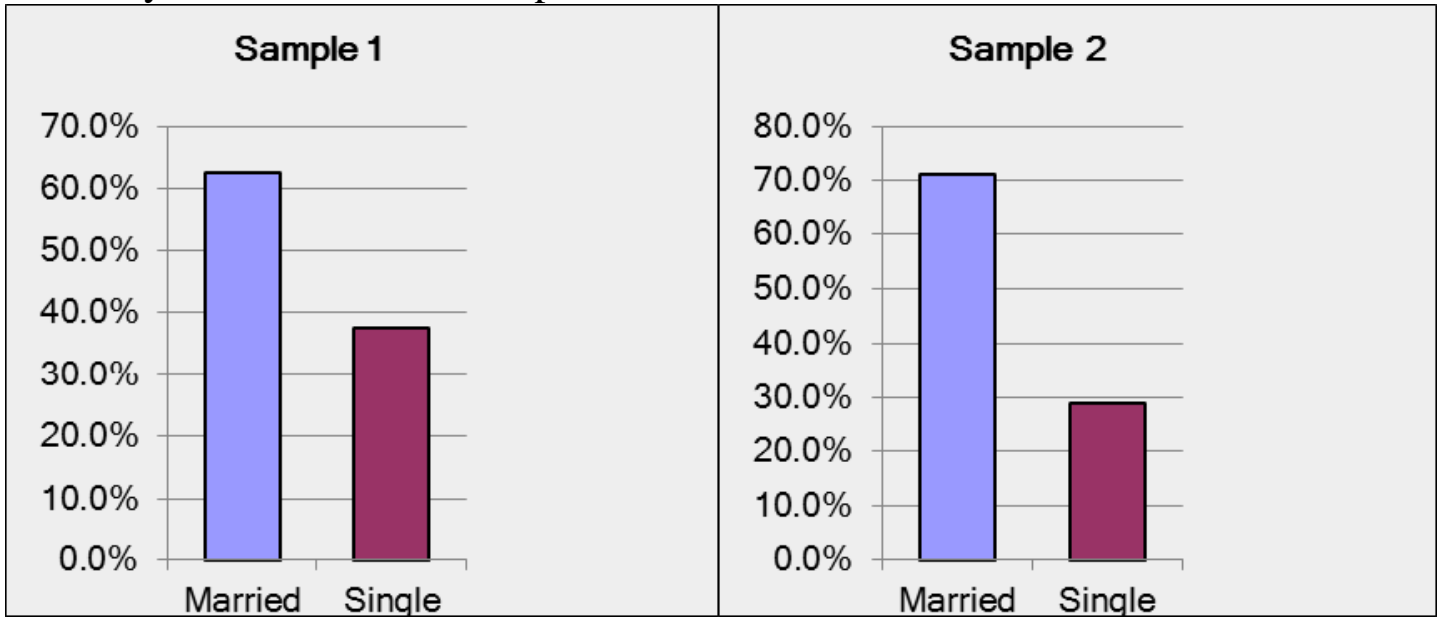

The overwhelming majority of participants were married.

Figure IVI: Gender

What is your gender?

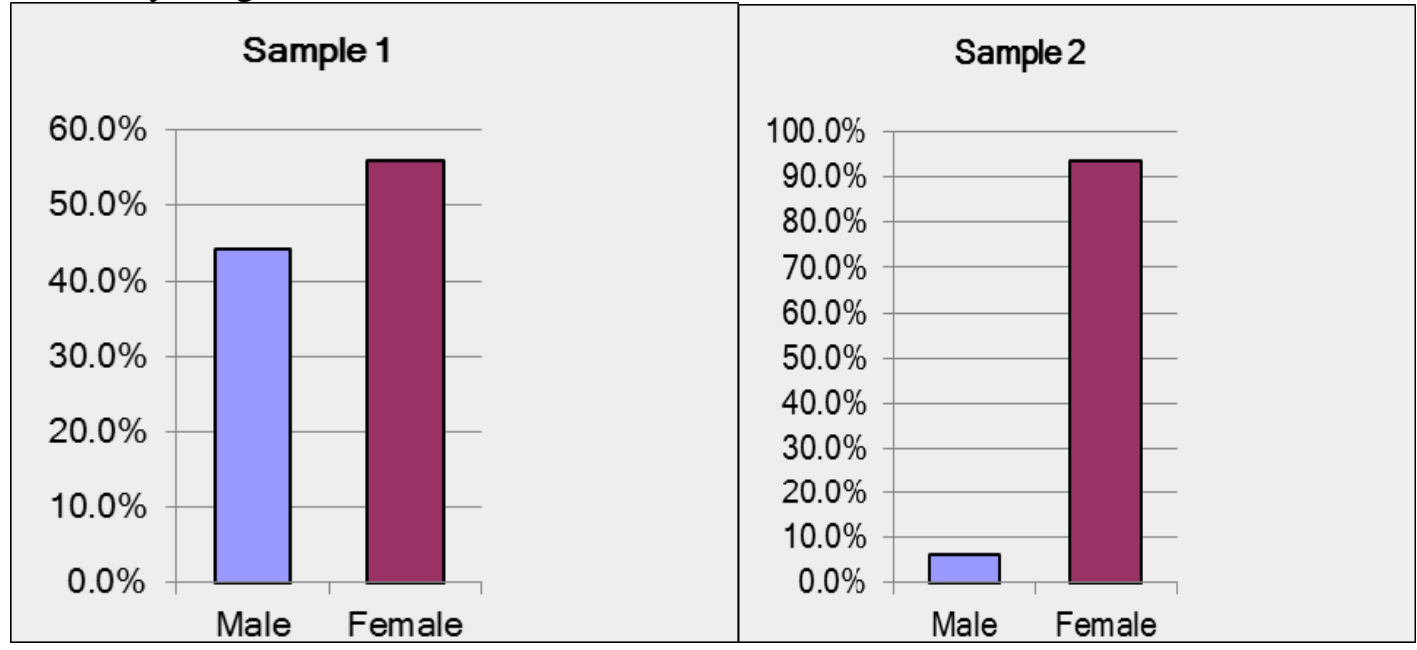

There was a significant overall difference in male teacher populations of both samples. 
Figure V: Overall Burn-Out Scores for Both Samples

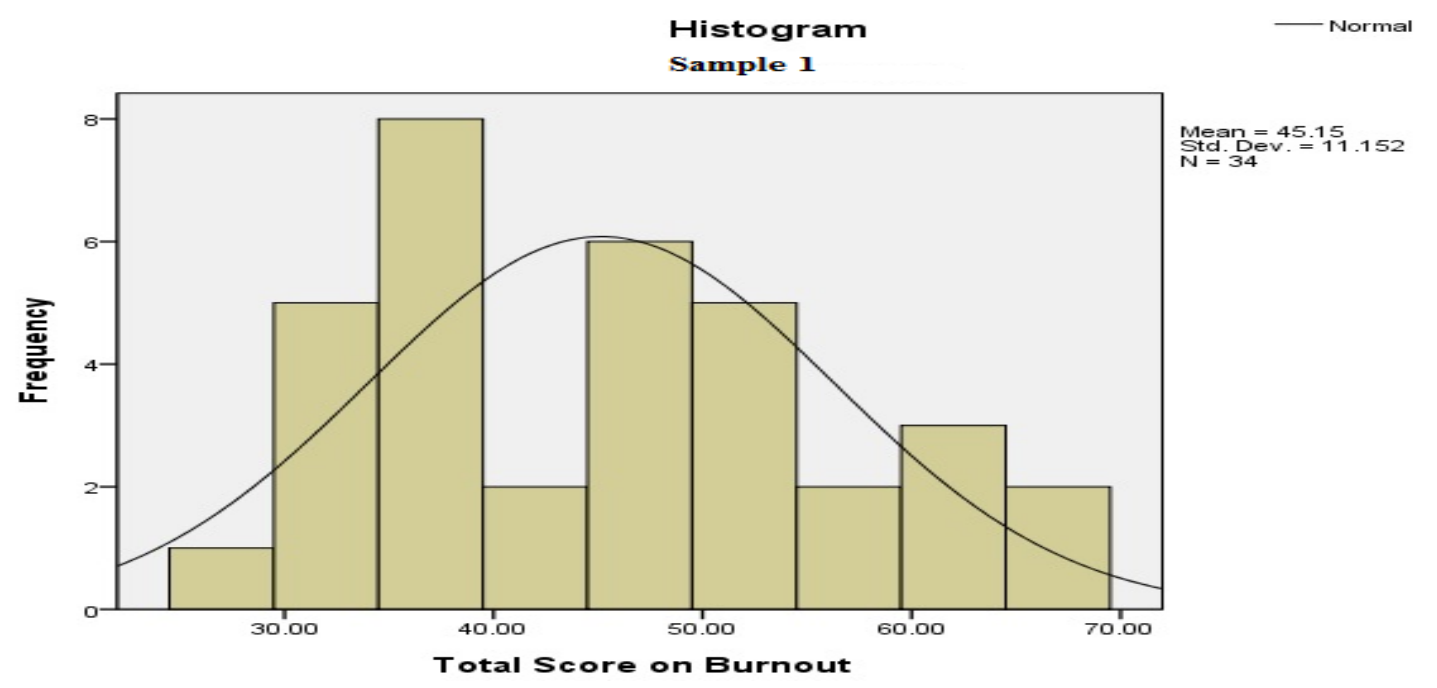

The total burn-out scores on this frequency curve for sample one indicate a slight positive skew. In other words, the mean of total burn-out scores was slightly less for sample one than a normal curve. However, it is still normally distributed.

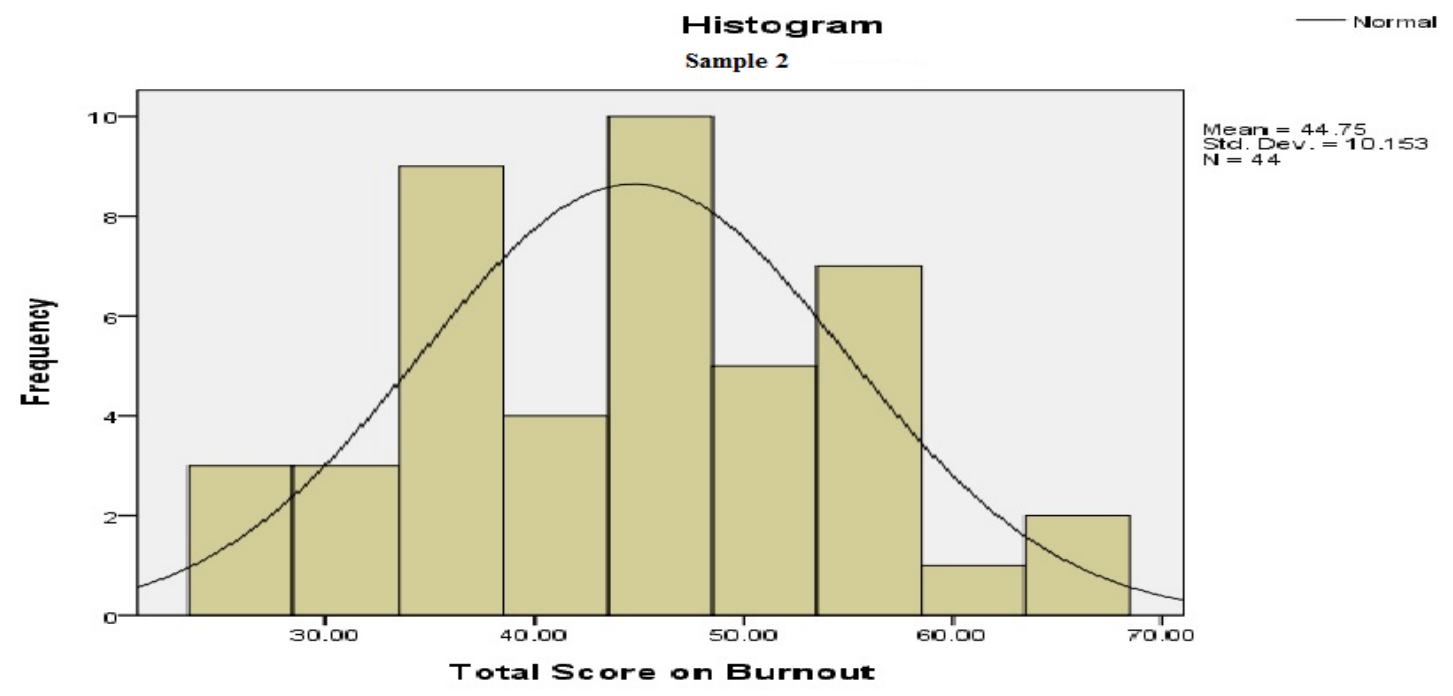

The total burn-out scores on this frequency curve for sample two indicate a relatively normal distribution. 
Figure VI: Q-Q PLOTS for Both Samples
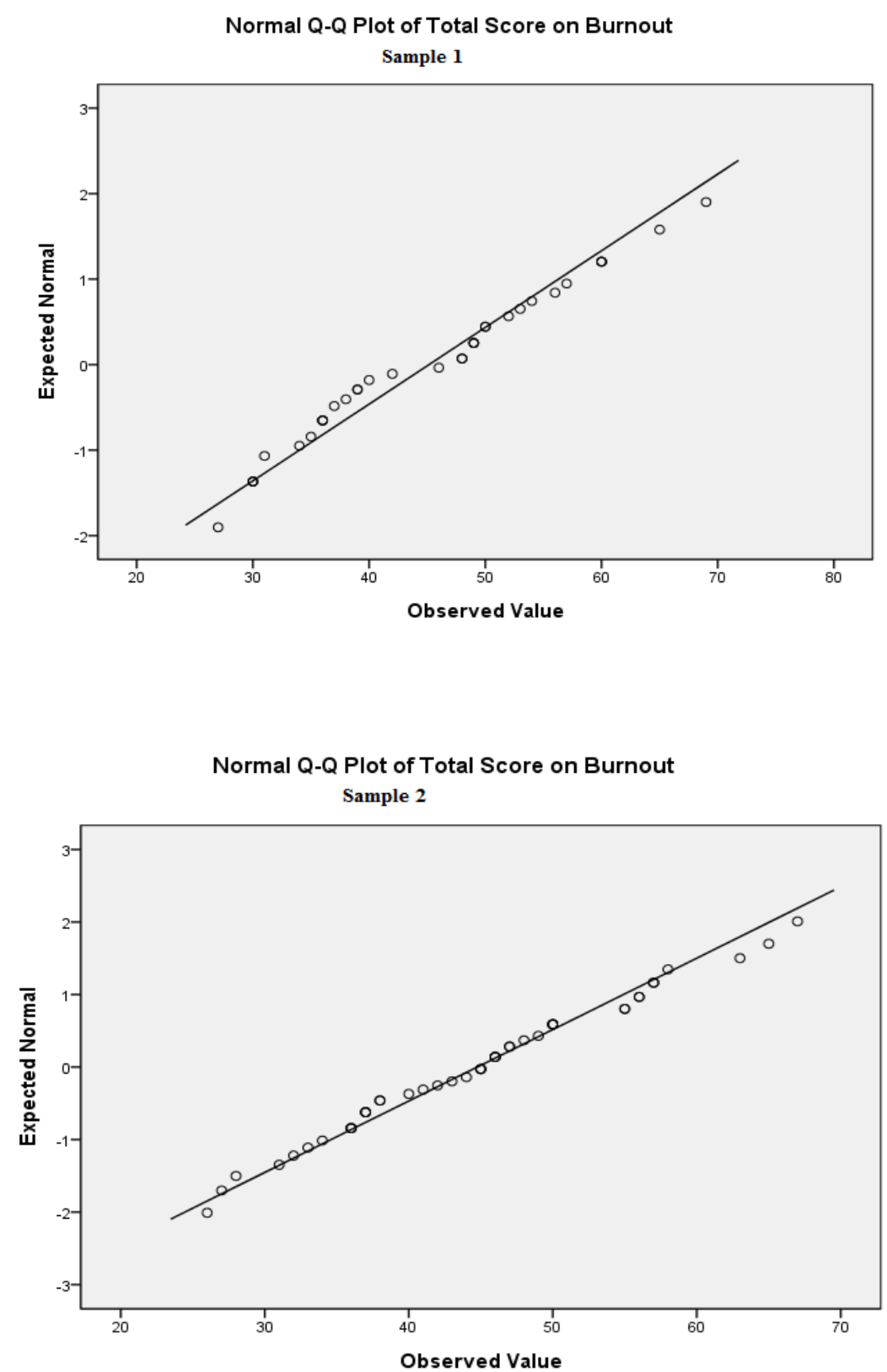

The linearity of the points on the following Q-Q plots suggests normality for both samples, without noticeable outliers. 
Figure IX: Total Burn-out Score by Sample Box Plot

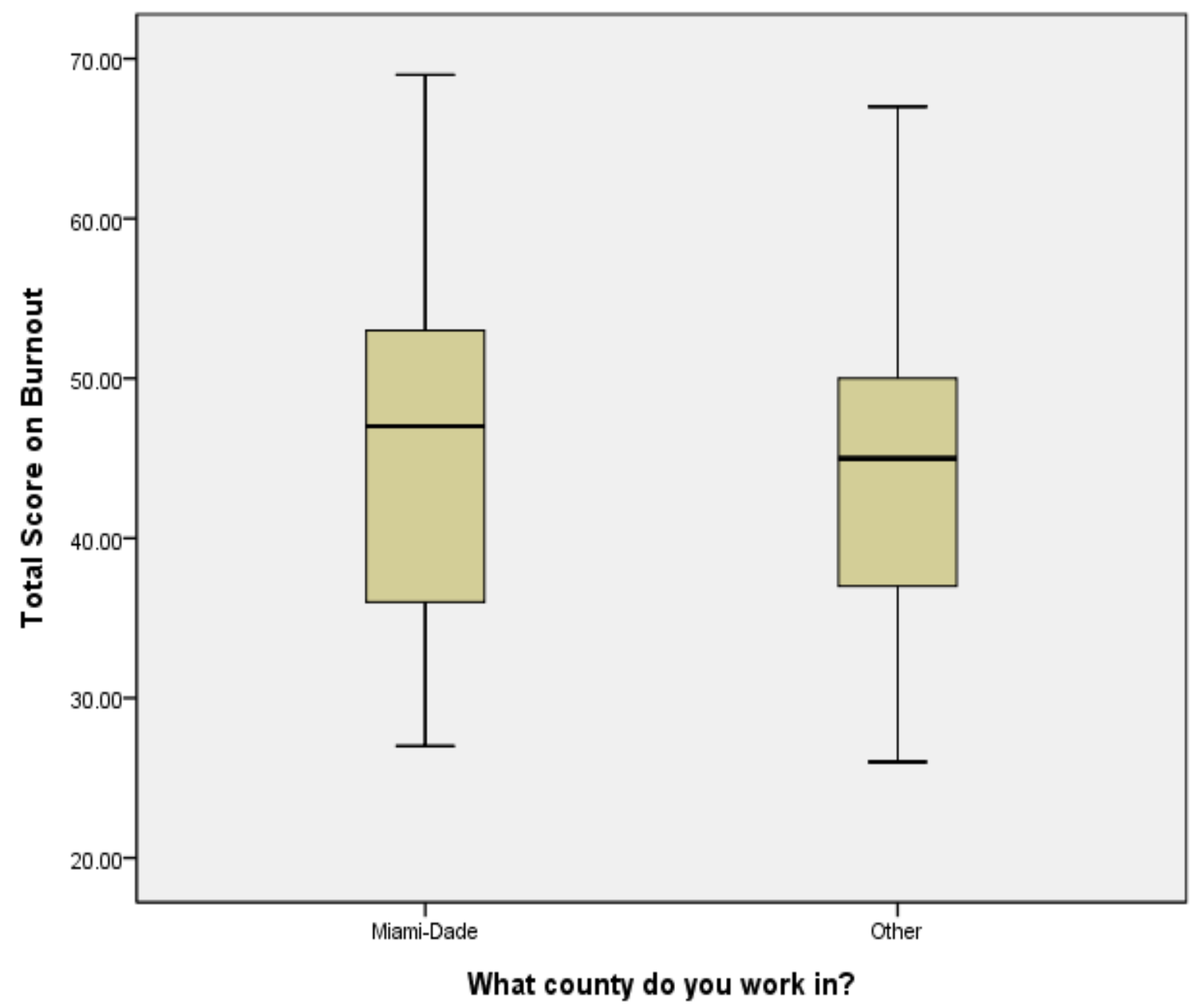

Although a significant difference was not found between the two populations, and the shape of the normal curves showed a slightly higher mean value for sample two; these box-plot and whisker plots show a higher median value for total burn-out scores within sample one, especially in the higher quartiles. 
TABLE IV: T-Test Group Statistics

\begin{tabular}{|c|c|c|c|c|c|}
\hline & & $\mathrm{N}$ & Mean & Std. Deviation & $\begin{array}{c}\text { Std. Error } \\
\text { Mean }\end{array}$ \\
\hline Total Score & Sample One & 34 & 45.1471 & 11.15220 & 1.91259 \\
\hline on Burn-out & Sample Two & 54 & 44.7500 & 10.15261 & 1.53056 \\
\hline
\end{tabular}

The mean for both samples only differed by .397, which is not significant.

TABLE V: Levene's Test

Independent Samples Test

\begin{tabular}{|c|c|c|c|c|c|c|c|c|c|c|}
\hline & \multicolumn{2}{|c|}{$\begin{array}{c}\text { Levene's Test } \\
\text { for Equality of } \\
\text { Variances } \\
\end{array}$} & \multicolumn{7}{|c|}{ t-test for Equality of Means } \\
\hline & & \multirow[b]{2}{*}{$\mathrm{F}$} & \multirow[b]{2}{*}{ Sig. } & \multirow[b]{2}{*}{$\mathrm{t}$} & \multirow[b]{2}{*}{ df } & \multirow{2}{*}{$\begin{array}{l}\text { Sig. (2- } \\
\text { tailed) }\end{array}$} & \multirow{2}{*}{$\begin{array}{c}\text { Mean } \\
\text { Differe } \\
\text { nce }\end{array}$} & \multirow{2}{*}{$\begin{array}{l}\text { Std. Error } \\
\text { Difference }\end{array}$} & \multicolumn{2}{|c|}{$\begin{array}{l}\text { 95\% Confidence } \\
\text { Interval of the } \\
\text { Difference }\end{array}$} \\
\hline & & & & & & & & & Lower & Upper \\
\hline \multirow{6}{*}{$\begin{array}{l}\text { Total } \\
\text { Score } \\
\text { on Burn- } \\
\text { out }\end{array}$} & Equal & \multirow[t]{6}{*}{1.155} & \multirow[t]{6}{*}{.286} & \multirow[t]{3}{*}{.164} & \multirow[t]{3}{*}{76} & \multirow[t]{3}{*}{.870} & \multirow[t]{3}{*}{.39706} & \multirow[t]{3}{*}{2.42000} & & 5.2169 \\
\hline & variances & & & & & & & & 4.4227 & 0 \\
\hline & assumed & & & & & & & & 8 & \\
\hline & Equal & & & \multirow[t]{3}{*}{162} & \multirow[t]{3}{*}{67.543} & \multirow[t]{3}{*}{.872} & \multirow[t]{3}{*}{.39706} & \multirow[t]{3}{*}{2.44962} & - & 5.2857 \\
\hline & variances not & & & & & & & & 4.4916 & 9 \\
\hline & assumed & & & & & & & & 7 & \\
\hline
\end{tabular}

On the Levene's Test for Equality Variances we can see that the significance level (.286) is much higher than .05. Thus there is not a significant difference between population variances, and this assumption was met. 


\section{CHAPTER V \\ DISCUSSION}

The data related to this study did not affirm that there was a significant regional difference between M-DCPS and other Florida counties regarding over-all burn-out levels. Therefore, the following conclusions can be made regarding the main research questions of this study:

1. There were no significant stressors unique to M-DCPS teachers.

2. Administrators supported music programs in M-DCPS at comparable levels to other Florida counties. Although the data would lead us to believe that sample two had more issues with administration.

3. Results related to the accountability movement question were almost identical in both populations. Although these results were positively skewed, meaning all participants found this issue to placing additional burdens on music programs, results seemed to indicate slightly more stress among members of the "other counties” sample.

While there was not a significant difference in over-all burn-out scores, sample one did show a significant difference related to survey item number nine (I find myself not wanting to see certain classes/or students), where the M-DCPS sample showed a $25.3 \%$ higher level of agreement; this would lead us to believe that sample one either had more challenging students, or less of a tolerance for student discipline issues. Also, interesting was self-reported responses to statement 14 (I feel that I am burned-out as a teacher) which were more negatively skewed for sample two, meaning that sample 1 felt slightly more burned-out when self-reporting. 
There were some unexpected results/trends regarding how sample one compared to other Florida teachers mostly related to the difference of age ranges. The majority of Florida teachers in this study were over the age of 41, many of whom were over 51 and soon set to retire; this was overwhelmingly true for sample one where $61.8 \%$ of the teachers in this sample where over 51. Sample two was a lot more balanced in age ranges. Also, many teachers listed their uncertainty about job security in the coming years a stressful area of their lives, as art and music programs are increasingly being threatened by political/economic conditions.

\section{Qualitative Self-Reported Perceptions}

The final two questions of the survey were meant to solicit insights that may not have been revealed through previous, relatively closed questions. A few interesting issues were conveyed by participants, such as stress caused by teacher salaries, the balance between work and personal life, and the need for equal respect given to the music education vocation. The most prevalent of these issues for the Florida workforce seemed to be related to a lack of proper remuneration, a lack of administrative support, and uncertainty about job security. Full answers to these final two questions can be found in Appendix D.

\section{Reducing the Effects of Stress and Burn-out}

\section{Approaches to Lifestyle Changes}

According to Maslach there are three main areas on which to focus to lessen the effects of burn-out: our-selves, other people, and our institutions. The following three paragraphs summarize her recommendations. Intrapersonal knowledge is essential in order to solve issues related to burn-out; to avoid them we must self-examine, for 
example by starting a stress journal and examining it every two weeks to look for patterns. This may help achieve another of Maslach's main tenants: "working smarter not harder.”

Maslach recommends constantly altering one’s methods, experimenting with different approaches, and "shifting gears." This is why engaging in continuous learning, attending conferences, enrolling in workshops, getting an advanced degree, staying up to date by following research, newsletters, etc. is so important (see also Preston, 2004). Various websites related to music education may be of assistance as well: http://www.menc.org/, http://acdonline.org/cj/, www.musick8.com/, and www.aosa.org.echo/default.asp (Preston, 2004).

Maslach also endorses setting goals one may reasonably expect to attain. Setting unrealistic goals may lead to feeling unaccomplished. She advises us to "break-away" from situations, by taking a mini-break (counting to 10, breathing, and doing imagery exercises) or a major break (going out to lunch, visiting with a colleague, or reading a book). Maslach's next two suggestions are linked: do not take work home, and do not take things personally. Work must be left at work, and interpersonal conflicts must be looked at objectively, so that one does not keep re-experiencing them.

Aside from Maslach's recommendation, taking care of oneself physically is also important in managing daily challenges. According to a recent study, however, only $28 \%$ of workers self-reported themselves as having excellent health, and $48 \%$ of the study participants report positively to having health issues (Families and Work Institute, 2008); $48 \%$ of music teachers in this study reported having some health issues. As a priority, taking care of one-self properly too often lags behind other life/work demands and 
accompanying pressures. This is especially true for music teachers who are expected not only to fulfill regular life and work demands, but also to lead after-school groups and extracurricular activities. Educators must learn to make this "work/life fit,” which means taking time preparing well-balanced meals, drinking plenty of water, exercising, and getting a proper amount of sleep (Families and Work Institute, 2008). There are many online resources, (http://www.mypyramid.gov/, www.mindtools.com/, http://www.heart.org/HEARTORG/, www.nhlbisupport.com/BMI/ , or http://www.cdc.gov/) that may assist. Maintaining health through exercise can also help ensure the energy required to teach music effectively, relieve stress, and give one time to de-stress away from school (Preston, 2004). Aerobic exercise is particularly well-suited for these purposes.

\section{Workplace Changes}

According to Leiter and Maslach (1997), there are six possible journeys to take when one is looking to avoid burn-out. All of these journeys are related to "mismatches" faced during the course of a career, such as feeling there is too little time to accomplish all that is necessary, or feeling a sense of efficacy in one’s profession after all one contributes. The "guidepost" for these journeys are "a sustainable workload, feelings of choice and control, recognition and reward, a sense of community, fairness-respect and justice, and meaningful and valued work” (Leiter \& Maslach, 1997).

There are, according to Leiter and Maslach (1997), two major areas to consider: “creating harmony between people and their jobs and changing the job environment as well as the people.” These changes may be difficult to attain if individuals feel undervalued. Maslach (1982) suggests seeking, rather than waiting for, praise. She 
proposes the following language: "I would appreciate knowing what you like about my work, as well as what you (dislike).” Such a request may be done at various times, such as during an end-of-the-year evaluation meeting with one’s administrator. As for creating more harmonious workplace relationships and environments, one could try communicating with the administration about its vision for the music program, speaking at faculty meetings, being a teacher (not just a "music teacher"), volunteering for committees, responding promptly to administrative needs, not taking unnecessary personal days, and involving administration in the music program (Preston, 2004).

Time management is important, but socialization with coworkers is also important in order to reduce workplace stress. According to the Families and Work 2008 report on the state of health in the American workforce, the American worker is experiencing a “time famine” (Families and Work Institute, 2008). For example, 11 participants in my study made comments related to not having enough time to get everything done. There are numerous approaches that may be taken to alleviate the pressures of not having enough time, such as delegation of tasks; taking such actions can possibly empower others by providing them with training, a sense of trust, and acknowledgment (Cedoline, 1982). Moreover one can also mentor, utilize organizational list, learn new technologies for productivity, and solicit help from family and friends.

\section{Conclusion}

Overall findings of this study revealed three significant demographic differences between populations in that sample one was older (especially in the over 51 age ranges), had higher levels of education, and had more male teachers. Additionally, sample one had a much higher positive response to item number nine of the survey (I find myself not 
wanting to see certain classes/or students) which could indicate one of two things, maybe sample one is less tolerant of student discipline issues, or maybe this population has a more challenging student body to cope with. However, these results could have been affected because of the significant differences in demographics of both samples, thus this study should be replicated.

Overwhelmingly frequent self- reported responses to the last two open-ended questions were related to a lack of administrative support, job security, and having enough personal funds. Twelve comments concerned stress caused by a lack of administrative support, 10 comments related to stress caused by a lack of job security, and six comments addressed stress caused by a lack of personal funds. In another study related to “Music Teacher Retention and Attrition”, Madsen and Hancock (2002) had respondents that wrote similar comments with similar percentages (37\% were concerned with administrative support, and nine percent were concerned with financial issues). However, job security was not brought up in that study. The prevalence of these written statements is understandable as administrators are judged by their school's performance on the FCAT, and increasingly the arts have been devalued in this climate of accountability. Research suggests that a lack of administrative support (music is not an FCAT-tested subject) has affected the manner in which music teachers view their profession (Mancini's thesis as cited in DeLorenzo, 1992, Karge, 1993, and Krueger, 2000).

Year after year, publicly employed music educators have faced cutbacks that the current economic has only magnified. Most alarming is that some study participants implied that they would retire earlier due to the fear of losing their retirement benefits. 
Local governments have "rolled back salaries, frozen scheduled pay increments, and used back-to-work legislation to counter public strikes...” and “...increased workloads exacerbating the impact of reduced compensation" to the point that these employees are facing financial immobility (Leiter \& Maslach, 1997). 


\section{LIST OF REFERENCES}

Allsup, R. E., (2005). Stress and the music teacher preventing burnout. Teaching Music, 12, (5), 50.

Aronson, E., Kafry, D. \& Pines, A. (1981). Burnout: from tedium to personal growth. New York, NY: Free Press.

Blazer, C., (2010). Information capsule: Teacher burnout. Retrieved March 6, 2011, from http://www.dadeschools.net/employees/TEACHER_BURNOUT.pdf

Cedoline, A. J., (1982). Job Burnout in Public Education. New York, NY: Teachers College Press.

Dworkin, G. D. (1987). Teacher Burnout in the Public Schools. Albany, NY: State University Press of New York Press.

DeLorenzo, L. C. (1992). The perceived problems of beginning music teachers. Bulletin of the Council of Research in Music Education, 113, 9-25.

Efland, A. D. (1983). Art Education during the Great Depression. National Art Education Association, 36(6), 38-42.

Families and Work Institute. (2008). National study of the changing workforce: The state of health in the American workforce. Retrieved February 7, 2011, from http://www.familiesandwork.org/site/research/reports/HealthReport.pdf

Farber, B. A. (1983). Stress and Burnout in the Human Service Professions. Elmsford, NY: Pergamon.

Guglielmi, R. S., \& Tatrow, K. (1998). Occupational stress, burnout, and health in teachers: A methodological and theoretical analysis. Review of Educational Research, 68 (1), 61-99.

Gudwin, D. M., \& Salazar-Wallace, M. D. (2010). Mentoring and coaching: A lifeline for teachers in a multicultural setting. Thousand Oaks, Calif: Corwin Press.

Hamann, D. L., \& Gordon, D. G., (2000). Burnout an occupational hazard. Music Educators Journal, 87 (3), 34-39.

Hancock, C. \& Madsen, C. K. (2002). Support for music education: a case study of issues concerning teacher retention and attrition. Journal of Research in Music Education, 50 (1), 6-19. 
Karge, B. D. (1993, April). Beginning teachers: In danger of attrition. Paper presented at the annual meeting of the American Educational Research Association, Atlanta, G.A.

Krueger, P.J. (2000). Beginning music teachers: Will they leave the profession? Update: Applications of Research in Music Education, 19(1), 22-26.

Leiter, M. P., \& Maslach, C. (1997). The Truth About Burnout: How Organizations Cause Personal Stress and What to do About it. San Francisco, CA: Josey Bass Inc.

Maslach, C. (1982). Burnout-the cost of caring. Englewood, NJ: Prentice-Hall.

McGrory, K., \& Samuels, R. (2009, October 5). Miami-Dade school teachers get a long awaited pay raise. Miami-Herald. Retrieved October 14, 2010, from http://infoweb.newsbank.com.ezproxy.fiu.edu/iwsearch/we/InfoWeb?p_field_base-0=\&p_text_base-0=MiamiDade+school+teachers+get $+a+$ long + awaited+pay+raise\&f_submit_search=Searc h\&p_params_base- $0=\& p \_b o o l \_b a s e-1=$ and \&p_field_base- $1=\& p \_t e x t$ base$1=\& p \_p a r a m s \_b a s e-1=\& p \_b o o l \_b a s e-2=$ and \&p_field_base2=YMD_date\&p_text_base-2=past+2+years\&p_params_base2=\%0D\%0AB\%0D\%0AE\%0D\%0A\&p_sort=YMD_date\%3AD\&p_product=AW

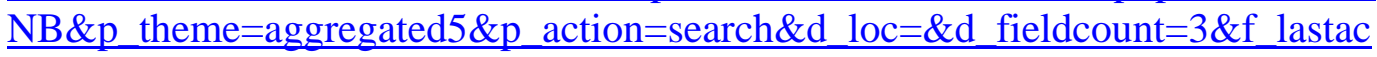
$\underline{\text { tion }=\text { explore } \& f \text { dateparams }=\mathrm{B} \% 2 \mathrm{CE}}$

Office of Assessment, Research, and Data Analysis. (2008, October). Student membership by race/ethnicity. Statistical abstract (Student and community demographics). Retrieved September 28, 2010, from http://drs.dadeschools.net/StatisticalAbstract/sa0708.pdf

Pines, A., \& Aronson, E. (1988). Career Burnout. New York, NY: The Free Press.

Preston, T., K. (Ed.). (2004). Teacher to teacher: A music educator's survival guide. Reston, VA: The National Association for Music Education.

Scheib, J.W., (2003). Role stress in the professional life of the school music teacher: A collective case study. Journal of Research in Music Education, 51 (2), 124-136. 
APPENDIX A

E-mail sent to 50 randomly selected M-DCPS principals 


\section{Dear (Principal)}

My name is Luciano Alberto; I am currently conducting a study on general elementary music teacher burnout in Florida for my master's degree from Florida International University. This study will compare a sample of music teachers from Miami-Dade County Public Schools to teachers from the rest of the state. I am writing you to inform you that I have randomly selected your school's music teachers for participation in this study. However, before proceeding I require your permission to e-mail your music teacher(s) my totally confidential survey. If for some reason you object to your school's music teachers participating, or have additional information about your music teachers you would like to provide me please e-

mail me at Lalberto@dadeschools.net by January $14^{\text {th }}$, as this is my target date for emailing this survey. Otherwise I will assume that you do not object to their participation.

Thank You,

Luciano Alberto

P.S. Attached will see my approval for this study from M-DCPS. 


\section{APPENDIX B}

E-mail sent to M-DCPS general elementary school music teachers 


\section{Dear Music Teacher}

My name is Luciano Alberto, I am currently conducting a study on general elementary music teacher burnout in Florida for my master's degree from Florida International University. This study will compare a sample of music teachers from Miami-Dade County Public Schools to teachers from the rest of the state. I am writing you to inform you that I have randomly selected you for participation in this study. This is a totally confidential and voluntary survey that would only take you between three to six minutes to complete. If you decide to participate in this study, you will be completing a survey I will be giving you today. Remember, this is completely voluntary. It is expected that this study will benefit music education in Florida by disclosing music teachers' levels of work related stress that could lead to school districts addressing burnout issues that could ultimately lead to attrition in this area of education.

If you decide to participate in this study, you may access the survey by clicking on this secure link to the survey that is secured using SSL security: http://www.surveymonkey.com/s/WX3T8MN

Remember, this is completely voluntary; you can stop participation at any time but I would really appreciate your participation. There are no foreseeable risks or benefits to you for participating in this study. It is expected that this study will benefit music education in Florida by disclosing music teachers' levels of work related stress that could lead to school districts addressing burnout issues that could ultimately lead to attrition in this area of education. You will remain anonymous. In any sort of report we might publish, we will not include any information that will make it possible to identify you as a subject. Research records will be stored securely and only the researcher will have access to the records. Your records may also be reviewed for audit purposes by authorized University or other agents who will be bound by the same provisions of confidentiality.

If you have any questions about the study, please do not hesitate to ask me at (xxx) 0819, or e-mail me at Lalberto@dadeschools.net.

\section{Thank You-Luciano Alberto}

P.S. Please feel free to forward this to your itinerant music teacher if you have one. 


\section{APPENDIX C}

On-line survey via SurveyMonkey 
1. I understand that this survey is totally voluntary and I can stop taking at any time if I choose. Do you consent to taking this survey?

E I understand that this survey is totally voluntary and I can stop taking at any time if I choose.

Do you consent to taking this survey? Yes, I would like to continue to the survey.

c No thank you.

2. 1.There are certain aspects related to my schools preparation for the Florida Comprehensive Assessment Test (FCAT) that take away from my program (e.g., student pull out, planning time being taken for FCAT activities, intervention I must teach, etc.).

c 1. There are certain aspects related to my schools preparation for the Florida Comprehensive Assessment Test (FCAT) that take away from my program (e.g., student pull out, planning time being taken for FCAT activities, intervention I must teach, etc.). Agree

c Somewhat Agree

E Neither Agree nor Disagree

c Somewhat Disagree

E Disagree

Other (please specify)

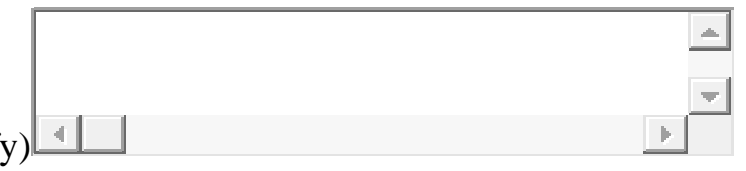

3. I feel that my school's administration supports/values my program.

c I feel that my school's administration supports/values my program. Agree

E Somewhat Agree

c Neither Agree nor Disagree

c Somewhat Disagree

c Disagree

Other (please specify)

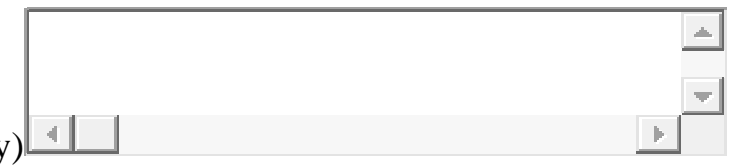

4. I have difficulty facing another workday when I wake up in the morning.

E I have difficulty facing another workday when I wake up in the morning. Agree

c Somewhat Agree

c Neither Agree nor Disagree

c Somewhat Disagree

c Disagree

Other (please specify)

\section{I spend time at my school performing non-music related duties (e.g., reading} tutoring, supervision duty, etc.).

E I spend time at my school performing non-music related duties (e.g., reading tutoring, supervision duty, etc.). Agree

c Somewhat Agree

c Neither Agree nor Disagree

c Somewhat Disagree

c Disagree 
Other (please specify) +1

6. My job has made me unsympathetic towards others.

c My job has made me unsympathetic towards others. Agree

c Somewhat Agree

c Neither Agree nor Disagree

c Somewhat Disagree

c Disagree

Other (please specify)

7. I feel physically drained at the end of the workday.

E I feel physically drained at the end of the workday. Agree

c Somewhat Agree

c Neither Agree nor Disagree

c Somewhat Disagree

E Disagree

Other (please specify)

8. I have too much of a workload on a weekly basis.

E I have too much of a workload on a weekly basis. Agree

c Somewhat Agree

c Neither Agree nor Disagree

c Somewhat Disagree

c Disagree

Other (please specify) $4 \square$

9. I feel that I have a balance between work and my home life.

E I feel that I have a balance between work and my home life. Agree

c Somewhat Agree

c Neither Agree nor Disagree

c Somewhat Disagree

c Disagree

Other (please specify)

10. I find myself not wanting to see certain classes/or students.

E I find myself not wanting to see certain classes/or students. Agree

c Somewhat Agree

c Neither Agree nor Disagree

c Somewhat Disagree

c Disagree 
Other (please specify)

11. I feel totally emotionally drained at the end of the workday.

E I feel totally emotionally drained at the end of the workday. Agree

c Somewhat Agree

c Neither Agree nor Disagree

E Somewhat Disagree

E Disagree

c Other

Other (please specify)

12. My work is affecting my health (headaches, high blood pressure, insomnia, etc. . ..).

E My work is affecting my health (headaches, high blood pressure, insomnia, etc. ...). Agree

c Somewhat Agree

c Neither Agree nor Disagree

c Somewhat Disagree

E Disagree

c Other

Other (please specify)

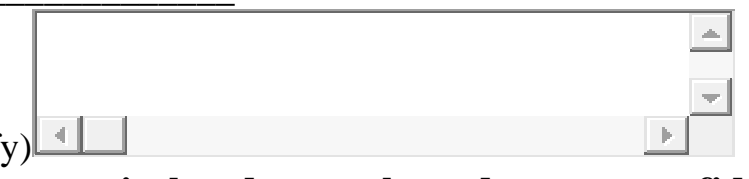

13. I feel that I am very isolated at work, and cannot confide in others.

c I feel that I am very isolated at work, and cannot confide in others. Agree

c Somewhat Agree

c Neither Agree nor Disagree

c Somewhat Disagree

E Disagree

c Other

Other (please specify)

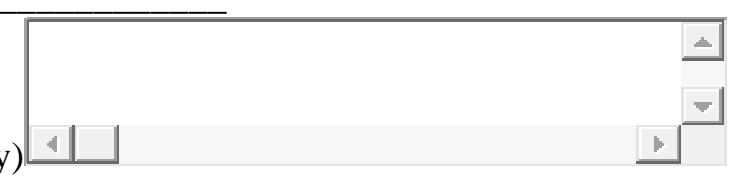

14. The politics of my school have hindered me at work.

c The politics of my school have hindered me at work. Agree

c Somewhat Agree

c Neither Agree nor Disagree

c Somewhat Disagree

c Disagree

c Other

Other (please specify) 4

15. I feel that I am burned out as a teacher.

c I feel that I am burned out as a teacher. Agree 
c Somewhat Agree

c Neither Agree nor Disagree

c Somewhat Disagree

c Disagree

c Other

Other (please specify)

\section{There are times when I am frustrated by trying to reach students who home} language is not English.

c There are times when I am frustrated by trying to reach students who home language is not English. Agree

c Somewhat Agree

c Neither Agree nor Disagree

c Somewhat Disagree

c Disagree

c Other

Other (please specify)

17. I find myself emotional exhausted on a weekly basis.

E I find myself emotional exhausted on a weekly basis. Agree

c Somewhat Agree

c Neither Agree nor Disagree

c Somewhat Disagree

c Disagree

c Other

Other (please specify)

18. Do you have school-aged children?

c Do you have school-aged children? Yes

c No

19. What is your highest level of education?

c What is your highest level of education? B.A ./B.S.

c Masters

c $\mathrm{PhD}$.

c Specialist degree

20. What is your age range?

c What is your age range? 21-30

c $31-40$

c $41-50$

c Over 51

21. Do you have your own classroom?

c Do you have your own classroom? Yes

c No

c Other 
22. Do you teach at more than one school?

[ Do you teach at more than one school? Yes

E No

c Other

Other (please specify)

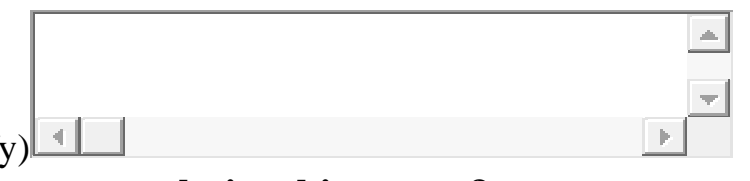

23. What is your current relationship status?

ᄃ What is your current relationship status? Married

c Single

24. What county do you work in?

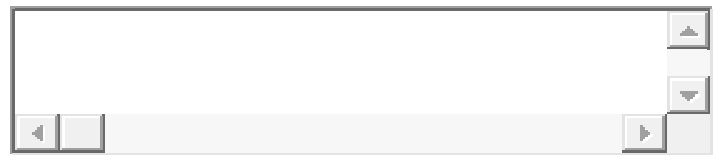

What county do you work in?

25. What is your major area of expertise?

E What is your major area of expertise? General Music

c Chorus

c Orchestra

E Band

[ Other

Other (please specify)

26. What is your gender?

c What is your gender? Male

c Female

27. How long have you been teaching?

[ How long have you been teaching? $0-5$ years

c 6-10 years

[ $11-20$ years

E More than 20 years; ___ If so how many years?

Other (please specify)

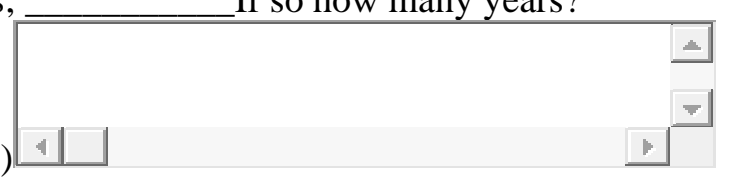

28. What is the most stressful area of your life right now?

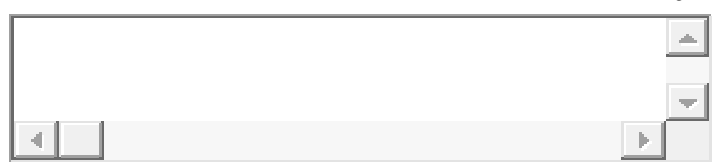

What is the most stressful area of your life right now?

29. Do you have any additional comments about your job as a music educator and your level of stress? 
APPENDIX D

Answers to Qualitative Survey Questions 
What is the most stressful area of your life right now?

\begin{tabular}{|c|c|}
\hline M-DCPS Sample & Other Counties Sample \\
\hline My job & Work, but it's fun stress \\
\hline Dealing with my principal & School. \\
\hline Finances & Putting off small things I need to do. \\
\hline $\begin{array}{l}\text { I love doing the music aspect of my job. It } \\
\text { relieves the stress of the non-music } \\
\text { elements of it }\end{array}$ & State of Florida's legislature and governor. \\
\hline Student's with behavior problems & $\begin{array}{l}\text { Chorus-no rehearsal time -4th grade } \\
\text { chorus. }\end{array}$ \\
\hline Dealing with student disruptions & Getting it all done well. \\
\hline Older parents & Administrative support \\
\hline I feel that my job is not important & Family Ties. \\
\hline My health & Work \\
\hline Money & Balance between work and home life. \\
\hline Uncertainty about having a job next year & My thesis \\
\hline Work & Home \\
\hline Marriage & My job \\
\hline $\begin{array}{l}\text { Are they going to cut music jobs next } \\
\text { year. I worry about the cutting out of } \\
\text { teachers. }\end{array}$ & After school programs \\
\hline My children getting ready for college & Finances \\
\hline $\begin{array}{l}\text { My room - it's freezing and constantly } \\
\text { makes me sick. Also, staying later after } \\
\text { school with chorus, catching up with work }\end{array}$ & $\begin{array}{l}\text { Scheduling balance between school and } \\
\text { life. }\end{array}$ \\
\hline
\end{tabular}




\begin{tabular}{|l|l|}
\hline $\begin{array}{l}\text { The schedule/planning during school and } \\
\text { keeping up with grade-book on the computer }\end{array}$ & Balancing work and family. \\
\hline $\begin{array}{l}\text { My teenage son, and having a feeling that } \\
\text { I need to retire before the state cuts my } \\
\text { job }\end{array}$ & My Children. \\
\hline Dealing with elderly parents & Finances \\
\hline Work and the fear of my job being cut & Finances \\
\hline Money & School duties other than music. \\
\hline $\begin{array}{l}\text { Parents who ask to have conferences quite } \\
\text { often to explain a "B" grade or behavior } \\
\text { issues }\end{array}$ & Home life \\
\hline Politics in school & Lunchroom duty! One hour per day. \\
\hline Not having a proper classroom & $\begin{array}{l}\text { My music room situation; I must keep the } \\
\text { instruments in the music-room but I am not } \\
\text { allowed to use it as a classroom because of } \\
\text { the noise. Instead I must use two separate } \\
\text { rooms and use the music-room as a storage } \\
\text { room. My parent is ill. }\end{array}$ \\
\hline & After school music clubs. \\
\hline & $\begin{array}{l}\text { Health } \\
\text { Ifoel like the kids are not getting enough } \\
\text { instruction. }\end{array}$ \\
\cline { 2 - 2 } & Planning \\
\cline { 2 - 2 } & School and Church music work. \\
\hline Preparing competitive chorus for MPA and having an eight month old. \\
\hline $\begin{array}{l}\text { Trying to find more time to compose } \\
\text { music }\end{array}$ & Work \\
\hline & Balancing work and home \\
\hline
\end{tabular}




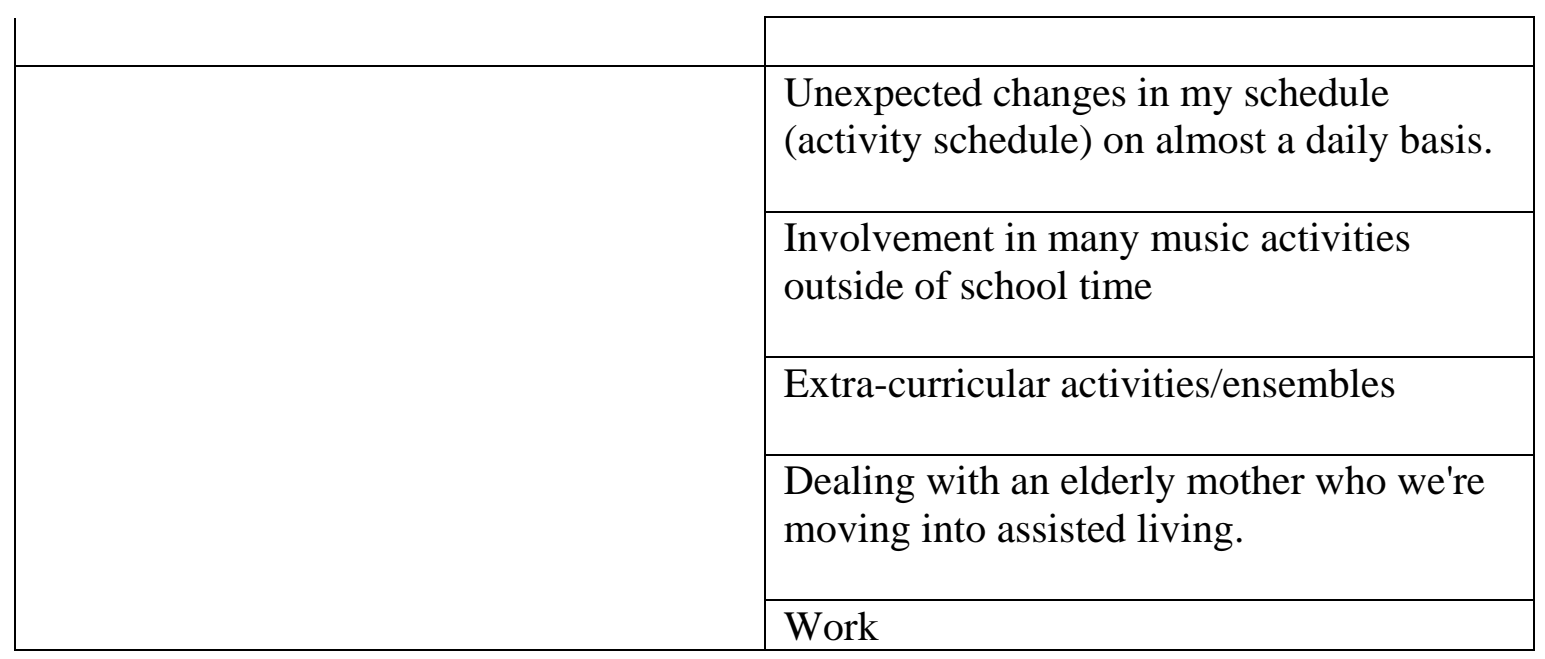

Do you have any additional comments about your job as a music educator and your level of stress?

\begin{tabular}{|l|l|}
\hline M-DCPS Sample & Other Counties Sample \\
\hline $\begin{array}{l}\text { Bring back real consequences for problem } \\
\text { students. }\end{array}$ & $\begin{array}{l}\text { I currently feel like a glorified babysitter } \\
\text { more than a music educator. Double classes } \\
\text { on a daily basis, even bigger groups when } \\
\text { other activity teachers pulled to substitute } \\
\text { for a classroom teacher, administrators who } \\
\text { tell me how to teach and how to do my } \\
\text { programs, and having to teach other things } \\
\text { (like structured P.E.) instead of music } \\
\text { during my activity time; this has made me } \\
\text { feel very de-valued this year. }\end{array}$ \\
\hline $\begin{array}{l}\text { The new expected bills from the state } \\
\text { legislature. }\end{array}$ & $\begin{array}{l}\text { I love teaching music. I get to share my } \\
\text { passion and learn from students daily. I am } \\
\text { so fortunate. } \\
\text { My job is fun! I keep it fresh by attending } \\
\text { as many workshops/in-services/conferences } \\
\text { as possible and by mentoring a fairly } \\
\text { steady stream of interns from the } \\
\text { University of South Florida. } \\
\text { It's stressful with a lack of resources and } \\
\text { creativity. }\end{array}$ \\
\hline For the most part I still very much enjoy & \begin{tabular}{l} 
I love being a music teacher. I just wish I \\
\hline
\end{tabular} \\
\hline
\end{tabular}




\begin{tabular}{|c|c|}
\hline teaching music. & $\begin{array}{l}\text { could teach and not worry about outside } \\
\text { stuff. } \\
\text { Music teachers don't get any/little opportunity } \\
\text { to gather, discuss what works and collaborate } \\
\text { on any regular basis in my district. }\end{array}$ \\
\hline $\begin{array}{l}\text { Combined classes may have saved my job } \\
\text { but handling } 40 \text { kids with recorders is } \\
\text { heavy. }\end{array}$ & I'm interested to see the results. \\
\hline $\begin{array}{l}\text { The harder part is never knowing if we will } \\
\text { have a job next year. }\end{array}$ & I receive no appreciation as a teacher \\
\hline $\begin{array}{l}\text { My elementary music program is not } \\
\text { respected at all. Children are taken out of } \\
\text { class every day, for counseling, for FCAT } \\
\text { practice, filing duties for their classroom } \\
\text { teachers. Also, as an elementary music } \\
\text { teacher, my job is threatened. My principal } \\
\text { was asking me about scheduling } 2 \text { classes } \\
\text { at once, and I said there's no need for } \\
\text { doubling up, there are open slots in the } \\
\text { schedule. She replied, "you know there are } \\
\text { a lot of people out there who want to work } \\
\text { and need to work. They would love this } \\
\text { job! I answered that I am fine with the } \\
\text { schedule; she's going to do what she wants } \\
\text { to do. }\end{array}$ & $\begin{array}{l}\text { I wish there were two of me ...enjoyable } \\
\text { and rewarding and draining, sometimes } \\
\text { frustrated. } \\
\text { The most stress in my personal life comes } \\
\text { from validating my program, e.g., trying to } \\
\text { make others-including administration see } \\
\text { why music is important and supportive of } \\
\text { the educational process at my school. } \\
\text { Music demonstration school process is } \\
\text { helpful in my avocation, as my school is a } \\
\text { Model Demonstration School. } \\
\text { When I get closer to a program(show), } \\
\text { that's when I feel the most stressed and run } \\
\text { down. }\end{array}$ \\
\hline Keeping my job!! I have no funds!! & I still love it. \\
\hline $\begin{array}{l}\text { I am disheartened at having to justify my } \\
\text { position to legislators, the school board(s), } \\
\text { and the general public. Music is an integral } \\
\text { part of the development of the human } \\
\text { brain; it facilitates learning at all levels, } \\
\text { especially among children and young } \\
\text { adults. Yet, it is overwhelmingly viewed as } \\
\text { superfluous, a "fluff" or a nicety, not the } \\
\text { necessity that it really is. I'm frustrated that } \\
\text { the general public doesn't view it as } \\
\text { essential. }\end{array}$ & $\begin{array}{l}\text { There is never enough time to meet all } \\
\text { standards, documenting reading and math } \\
\text { standards due to title1 funding for } \\
\text { elementary music. Not sure of how music } \\
\text { will survive the next round of } \\
\text { budget/teacher pay issues. }\end{array}$ \\
\hline $\begin{array}{l}\text { It's been a long road, but the journey has } \\
\text { been great as a music teacher. }\end{array}$ & $\begin{array}{l}\text { Get work done at school and don't bring it } \\
\text { home with you! This will help keep your }\end{array}$ \\
\hline
\end{tabular}




\begin{tabular}{|c|c|}
\hline & $\begin{array}{l}\text { fire burning. } \\
\text { One moment in a day often reminds me } \\
\text { that despite the many challenges ... I still } \\
\text { love being a teacher. I know I'm } \\
\text { contributing to music education positively. } \\
\text { I put a lot of stress on myself as I am a } \\
\text { perfectionist. Also, my schedule is getting } \\
\text { fuller by the week with new students } \\
\text { coming to our school classes are being } \\
\text { doubled. } \\
\text { Trying to get everything in and doing a } \\
\text { good job! Basically-I want my kids to love } \\
\text { music and remember what they have } \\
\text { learned in class. }\end{array}$ \\
\hline $\begin{array}{l}\text { Stress when classes are double, more than } \\
38 \text { students in a general music classroom. } \\
\text { No textbooks or materials available and I } \\
\text { have to spend my own money for supplies. }\end{array}$ & $\begin{array}{l}\text { More work is being put on teachers-less } \\
\text { help, parent abuse, media negativity. } \\
\text { This is the best job I've ever had. }\end{array}$ \\
\hline $\begin{array}{l}\text { My classes are too large-one class is over } \\
54 \text { students. This is extremely stressful and } \\
\text { not fair to the children as well. Class sizes } \\
\text { are too large. }\end{array}$ & $\begin{array}{l}\text { I'm thankful to have our student's } \\
\text { performing in all-state. Grateful for my job. } \\
\text { I have the greatest job in the world. } \\
\text { Overall, I love it despite the stress }\end{array}$ \\
\hline I love my job believe it or not. & $\begin{array}{l}\text { Dealing with administration is the most } \\
\text { stressful part of my job }\end{array}$ \\
\hline $\begin{array}{l}\text { Stress over the current political perception } \\
\text { of education. }\end{array}$ & $\begin{array}{l}\text { Should we start a teacher burn-out support } \\
\text { group? }\end{array}$ \\
\hline $\begin{array}{l}\text { Students being pulled-out and the constant } \\
\text { interruptions are very frustrating. }\end{array}$ & I love the kids they de-stress me. \\
\hline $\begin{array}{l}\text { The teacher allocations are ridiculous! We } \\
\text { keep getting cut and cut. According to my } \\
\text { principal in a K-8 there are no allocations } \\
\text { for any electives-she has to buy them! And } \\
\text { the emphasis on the FCAT is off the wall. } \\
\text { It's what drives the school, the schedules, } \\
\text { and the programs. The district mandates } \\
\text { intervention/tutoring classes but doesn't } \\
\text { provide the additional funds for tutors, } \\
\text { why? Then, we have to do it. What about } \\
\text { coaches and media specialist-why don't }\end{array}$ & $\begin{array}{l}\text { Only stressful when not supported by } \\
\text { administration or hindered by } \\
\text { administrator. } \\
\text { What to do about stress. }\end{array}$ \\
\hline
\end{tabular}




\begin{tabular}{|l|l|}
\hline they have to do the tutoring as well? & \\
\hline I love it. & $\begin{array}{l}\text { I've been moved 11 times in 23 years; in an } \\
\text { old kitchen while in the cold/wet weather, } \\
\text { up to } 9 \text { classes are in the eating area of cold } \\
\text { cafeteria-no walls-just filing cabinets, etc. }\end{array}$ \\
\hline $\begin{array}{l}\text { A principal who entertains parent } \\
\text { complaints and requests without first } \\
\text { checking with me. }\end{array}$ & \\
\hline \begin{tabular}{l|l} 
Administration does not support fine arts. \\
It's very difficult to work with minimal \\
funding. It's hard to justify taking groups to \\
MPA and festivals to a non-music \\
administration.
\end{tabular} & \\
\hline
\end{tabular}

